\title{
Product Life Cycle and Innovativeness: The Case of MENA
}

\author{
Uğur Aytun', Yılmaz Kılıçaslan² \\ Received:20/09/2017; Revised:14/11/2017; Accepted:08/12/2017
}

\begin{abstract}
One discussion in the literature of economics is the interaction between product life cycle and technological advancement. Firms may enjoy high profits and pay higher wages to the workers due to higher prices by introduction of new products in early stages and product developments in later stages. The aim of this study, by assuming that life cycle stage of a product represents its level of technology intensity, is to measure the innovative capabilities of selected benchmark and MENA countries by developing a maturity index, and then to see how MENA countries adapt themselves to relative maturity changes of products at the global level. Empirical findings using COMTRADE bilateral trade data for the period 1996-2013 showed that most of MENA countries' -especially Algeria and Turkey- adaptation performance fall in high- and low-tech industries. Moreover, adaptation pattern of MENA countries except for Egypt shows a decreasing trend in the highest growing products in the World trade. The performances of benchmark countries such as USA, South Korea, and Germany in terms of maturity adaptation were found to be increasing. We found that adaptation performance of MENA countries showed improvements in medium high- and medium low-tech industries mostly due to chemical and plastic industries in the last quarter. All these findings imply to call for policies that give incentive to create young products in high-tech and most demanded industries and to rejuvenate those already exist.
\end{abstract}

Keywords: product life cycle, manufacturing industry, innovation, MENA.

JEL Codes: F44; O12; O14; O30

\footnotetext{
${ }^{1}$ Department of Economics, Ahi Evran University, Kirsehir, Turkey. uguraytun@ahievran.edu.tr

2 Department of Economics, Anadolu University, Eskisehir, Turkey. ykilicaslan@ anadolu.edu.tr
} 


\section{Introduction}

Technological change with the introduction of ICT (Information and Communication Technologies) and removal of trade barriers over the past 35 years has led to substantial structural changes in both production and trade of developing and developed countries. Some late developing or developed countries, such as South Korea, Japan, and to a certain degree, China, started the transformation by establishing assembling industry. This, then, followed by creation of their own brands in young and technology intensive industries like automobile and electronics. However, some other countries, especially those in Africa and Middle East, specialized in the industries mostly based on resource and low technology insensitive industries. This paper addresses the question whether technological efforts and adaptation to product developments may contribute to the trade performance of countries.

Since Vernon (1966)'s seminal work on product life cycle, it is well known that all products have evolutionary process in global trade like human being. The process includes introduction, growth, mature, and decline in trade. Innovative activities vary across in these stages of product life cycle. For example, in early stages of the cycle, the activities are more intense and thus young products provide substantial gains to those who make these products because they are price-maker. Therefore, a country specializing on these products will develop its competitiveness, the degree to which a country can produce tradable goods while raising the income level of its citizens (OECD, 1992, p. 237). In the later stages, those focusing more on process innovation and investing in capital equipment may also keep their position in the market.

Based on the close relation between innovative efforts and life cycle of the product, in this paper, we aim to construct an innovativeness index by calculating maturity levels of manufactured products. This analysis allows us to see which products have upward or downward maturity trends at national and global scale. Moreover, this analysis sheds light on how countries adapt the innovativeness of their products relative to the global market for different technology intensive industries based on OECD (2011, pp. 5) manufacturing industry classification and for most-demanded products.

We estimate quadratic econometric models to calculate maturity index for each product for MENA countries and selected benchmark countries by using COMTRADE bilateral trade data for the period 1996-2013.

The paper is organized as fallows: In section 2, we discuss the underlying theoretical and empirical studies on the link between innovation and product life cycle. We introduce how we calculate the innovativeness index following Audretsch et al. (2012)'s methodology in section 3. Section 4 presents some empirical evidence for selected products, MENA and some industrialized countries. Finally, section 5 concludes and derives sector-level policy implications for MENA countries.

\section{Product life cycle and innovation}

The concept of "product life cycle" was first introduced by Jones in 1957. He explains the effect of "new products" on sales growth as follows (Gardner, 1986, pp. 5):

"There are compelling forces behind this drive for new products. There is life cycle that is characteristic of many -if not most-products. Since all products are 'new' at their outset, we can call it the basic life cycle for new products."

Life cycle of a product has five stages: introduction, growth, maturity, saturation, and decline. Levitt (1965) extended this approach that degree of newness and complexity of any product 
determines the length and slope of development stage. Producers, on the other hand, may delay later stages (maturity and decline) through product improvements and keep their products alive. Tallis and Crawford (1978, pp. 131) stated that only if innovative modifications are made, it is possible to claim that product reaches to the death stage. One can argue that there is an interrelation between life cycle of the product and innovative efforts. On the one hand, life cycle of the product determines the kind and magnitude of the innovative efforts, innovative efforts of entrepreneurs may affect the life cycle of the product on the other. For example, according to Abernathy and Utterback (1978, pp. 42-45), in the early stages, radical innovations are most likely to occur, thanks to science-based universities or entrepreneurially oriented financial institutions. As the industry matures, firms focus more on "formal" research and development investments that may include the process innovation and product differentiation through functional improvements. In fact, technological efforts are affected by the supply and demand conditions of the market. Stadler (1991, pp. 303), thereby, has modified this approach by emphasizing that in the search period for a new product, R\&D expenditures have fastest basic innovation rate in the "pre-innovation" market structure which is a type market between monopoly and perfect competition. As product matures over life cycle, R\&D efforts decreases because of limited technological opportunities. Klepper (1996, pp. 562-563) has also modelled the product life cycle model by examining entry and exit rates of firms and concluded that when industry is on the introduction stage, the entry precedes the exit and thus product innovation is to be high. This fallows with diminishing of the number of firms and product innovation rate. Finally, Audretsch (1987, pp. 302-304) empirically showed that innovation rate and labor skills affect positively the likelihood of being in the stage of "growth" and negatively "declining" or "mature" stage in U.S industries. The impact of capital-output ratio compared to innovation rate and labor skills has opposite effects on these stages.

Differently from the literature mentioned above, Vernon (1966, pp. 200) argued that the probability of creating new products is higher in developed countries because of high income and unit labor cost. The production location of new products would then shift to the developing countries due to low labor cost and not to lose the market. This argument based actually on Leontief's paradoxical findings about the trade patterns of US industries were found to be consistent with empirical evidence by several studies (Keesing, 1967; Gruber et al., 1967; Baldwin, 1971; Hirsch and Bijaoui, 1985; Lee and Stone, 1994). Keesing (1967, pp. 40-45) analyzed correlation coefficients between the international competitiveness and various variables such as R\&D expenditures, capital and labor skill requirement, and economies of scale and found strong evidence that R\&D activity is highly correlated with trade performance. Moreover, he confirmed that $\mathrm{R} \& \mathrm{D}$ is associated with scale and skill requirements and concluded that comparative advantages of US industries are primarily explained by the newly created products.

Gruber et al. (1967, pp. 23-30) also examined the link between the competitiveness of US exports and $R \& D$ expenditures assumed to be the proxy for the new product orientation. They found that there is a strong relation between the World share of US exports and R\&D expenditures in the industries having higher share of total US exports. However, they reached insignificant results when Germany and United Kingdom were used as denominator. This result was attributed to the similarity of export profiles of these countries. They found significant and positive coefficients in another exercise in which they normalized US exports with French and non-Europe exports whose innovational habits different from US.

Baldwin (1971, pp. 141-143) concluded that trained labor mostly engaging in research and development activities give temporary advantage based on technological opportunities in their industry level cross-section regression model where dependent variables were net US exports 
across trade partners (Canada, Japan, and Europe) and the World.

Hirsch and Bijaoui (1985, pp. 247-248) used trade, R\&D and size data of Israeli innovative firms in a regression analysis for period 1975-1981. They accepted the product life cycle hypothesis that the higher $\mathrm{R} \& \mathrm{D}$ intensity is associated with higher propensity to export. However, in their analysis, they didn't find a significant relation between firm size and export propensity. They explained this finding with the fact that every rational firm must reach certain domestic sales volume to make $R \& D$ profitable.

Lee and Stonae (1994, pp. 759-762) distinguished the product R\&D from process R\&D expenditures and used them as regressors in export model for two-digit eleven US manufacturing sectors for 1974-1978. Their panel data model findings controlling the fixed and time effects showed that both kind of technological efforts equally affect the export performance of industries.

According to Krugman (1979, pp. 265-266)'s simple general equilibrium model to formalize the life cycle theory, new industries systematically born in North and obtain quasi rents due to the monopoly power. These industries would then disappear because of cost competition with developing South. Finally, transfer of technology to South ends up this cycle.

Grosman and Helpman (1989, pp. 28-29) incorporated life cycle theory into endogenous growth theory. In their model, length of the life cycle, innovation and technology transfer are endogenously determined. As Krugman (1979) found, they concluded that if south accelerates the imitation rate of new products by widening resource base and developing learning activities, monopoly period of North would shorten. This will push the North to increase the innovation rate to keep living standards high by expanding the size and product development.

The two models above did not consider whether distinct effects take place between skilled and unskilled labor as the cycle evolves. By using similar modeling, Audretsch and Sanders (2007, pp. 2) found that entrepreneurs and skilled labor undoubtedly benefit from the new opportunities in the North.

\section{Data and methodology}

In this study, we use the United Nations' COMTRADE (2014) bilateral trade database. Harmonized System (HS) is so highly disaggregated (6 digit, 4954 sectors including manufacturing and agriculture for period 1996 to 2012) commodity classification that makes possible to analyze on almost single product level.

We used the approach developed by Audretsch et al. (2012) to measure the maturity level of products at global and national level. Before showing the new index, we introduce traditional life cycle models based on Audretsch (1987), Audretsch and Feldman (1996) and Sanders et al. (2007). These models generally regress the total sales against quadratic time trend. If sales variable is replaced by global export value of the commodity, then the equation to be estimated may be written as follows (Audretsch et al., 2012, pp. 5-6):

$$
\ln \left(\exp _{i t}\right)=\beta_{0}+\beta_{1} t+\beta_{2} t^{2}+\beta_{3} \ln \left(\exp _{t}\right)+\epsilon_{i t}
$$

In equation (1), $\exp$ represents the global export value and subscripts $i$ and $t$ denote product and time, respectively. For the right hand, $t$ and $t^{2}$ are used to determine the stage of product life cycle. $\exp _{t}$ is added to model to control for the global business cycle. Finally, $\epsilon_{i t}$ is the usual error term. After taking the derivative of equation (1) with respect to time, we can obtain the maturity index $\left(M_{i t}\right)$ of product $i$ for a given year (Sanders et al., 2007, pp. 10-11): 


$$
M_{i t}=\frac{\partial\left(\ln \left(\exp _{i t}\right)\right)}{\partial t}=\beta_{1}+2 \beta_{2} t
$$

According to equation (2), higher $M_{i t}$ value means less maturity of product $i$, or vice versa. Superiority of the index value calculated above is to be a time varying variable. Moreover, this index shows the rejuvenated products via technological developments or product differentiation.

We take this approach one step further in order to construct a maturity index for each tradable product of country $j\left(M_{i j t}\right)$ :

$$
\begin{aligned}
& \ln \left(\exp _{i j t}\right)=\gamma_{0}+\gamma_{1} t+\gamma_{2} t^{2}+\gamma_{3} \ln \left(\exp _{j t}\right)+\varepsilon_{i j t} \\
& M_{i j t}=\frac{\partial\left(\ln \left(\exp _{i j t}\right)\right)}{\partial t}=\gamma_{1}+2 \gamma_{2} t
\end{aligned}
$$

where $\exp _{i j t}$ is export value of product $i$ for country $j$ at time $t$. $\exp _{i t}$ in equation (3) controls for export fluctuations in country $j$, differently from (1) and (2).

By using equation (2) and (4) we can find world's and country $j$ 's maturity index value in period $t$, respectively. Dividing relative changes of these values each other proportionally and then taking weighted average for each technology category and for 150 high-share products, we can also see the adaptation degree of country in question to world market in terms of maturity change.

\section{Empirical Findings}

In order to examine the countries' innovativeness performance, we first selected 150 products as the best performers. These products were chosen with respect to both trade performance and maturity level. Specifically, we chose the products not only whose maturity is positive but also World trade shares are the highest in the last 5 years. The 150 youngest products based on 2013 maturity score and percentage maturity score change between 2009 and 2013, respectively, are presented in Table 1-2 in appendix. These products have little weight in world trade and more than half of them significantly consist of low-tech and/or medium-low tech products according to OECD $(2011$, pp. 5) classification. This finding is also consistent with Audretsch et al. (2012, pp. 7). They explain this finding with the fact that resource boom in 1990s led to considerable increase in trade volume and thus they seem to be rejuvenated. It is also questionable that moving toward an industry with low demand-low technological intensity spurs the development of a country. To overcome these problems, we decided to leave these products out and to consider those having high trade shares and positive maturity growth for the period 2009-2013. In Table 3 in appendix, we ranked the 150 products with highest five-year average trade share in global market based on maturity growth rate. As expected, more than two-thirds of these products have turned out to be medium high- and high-tech products. Note that 15 out of first 30 youngest products are medium high-tech and subject of value chains (Taymaz et al., 2011). This suggests that traditional sectors like automobile may be in the foreground in global market. We present global trade values of three different groups of 150 selected products in Figure 1. we aggregated global trade values of products of each table above and compare their trends. Figure 1 depicts that " 150 products with highest maturity value" (right axis) has steepest trend in last five years. The trend of "Total trade of 150 products with highest share and positive maturity growth" (left axis) is similar. "150 products with fastest maturity growth" (right axis) constitutes a relatively small share of World trade. In this study, we chose and examine "150 products with highest share and positive maturity growth". 
Figure 1: Total trade of 150 products with the highest share and positive maturity growth (left axis), 150 products with highest maturity value (right axis), 150 products with fastest maturity growth (right axis), 1996-2013

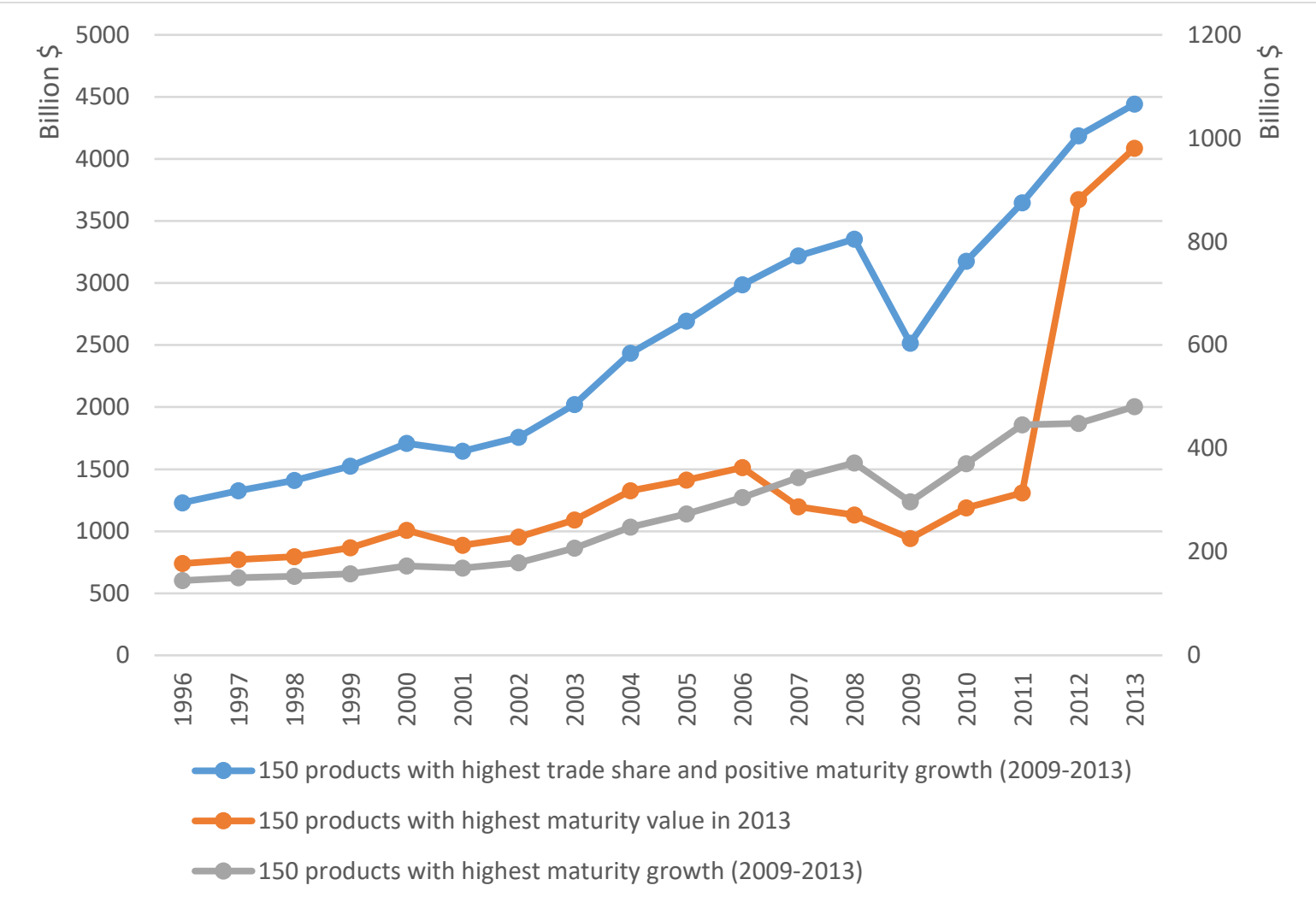

Source: Authors' calculations based on COMTRADE (2015) database.

\subsection{Twenty youngest products in the highest trade share group}

In the sections, we compare the MENA and benchmark countries' maturity performance trend with global market for 20 individual youngest products selected (Figure 1-20 in Appendix-B).

Overall implication of these figures is that US, Germany and South Korea sharply diverge from global market tendency in five products. The figures show that the maturity patterns of 8 products diverged from the World pattern in China. In other industries, despite of lags or leads, their maturity index patterns are pro-cyclical.

For MENA region, the first thing we must state that all MENA countries except for Turkey could not continuously managed to export all 20 youngest products over the period. Secondly, for last five or six years, these countries showed more divergent pattern than benchmark countries (US, Germany, South Korea, and China). In half of the number of products, on average, they formed scissor-shape with global curve. Among this group, Jordan is the best performer, having four out of seventeen of scissor-shapes. Jordan is followed by Algeria (4/9) and Oman (4/10). The worst performers among MENA countries are Saudi Arabia (9/15), Yemen (3/5), Turkey (13/20), and Egypt (10/15). In sum, MENA countries were not successful in adapting their product development to global trends.

\subsection{Technological categories and 150 highest share products}

In this section, we compare each skill and technological intensity and 150 most demanded products, by taking weighted average with total export shares. Our weighted average results are tabulated in the Table 1-5. To see the structural changes in countries and avoid business 
cycles such as 2001 and 2008, we split our sample into four periods, and then proportioned the relative maturity changes of countries to world. Furthermore, to see changes in the productive structures of countries not based on natural resources, we implemented the procedure again by excluding them in medium-high and medium-low tech industries since chemical (except pharmaceutical industry) and plastic industry is closely related with the petroleum industry.

Table 1 shows the results of the benchmark countries' high-tech industry analysis, which include aircraft, pharmaceuticals, office and computing machinery, radio, TV and communications equipment, medical precision and optimal instruments. Our results illustrate that adaptation values of China, Germany and US increased at the end of the period compared the beginning. South Korea, on the other hand, fade away its performance in this period. Among MENA countries, adaptation value of Algeria, Jordan, Kuwait, Morocco, Oman, Qatar, Tunisia, Turkey and Yemen decreased in the same period.

In the medium-high tech industries (electrical machinery, motor vehicles, chemicals excluding pharmaceuticals, railroad equipment and machinery equipment), China is the only country increasing the maturity adaptation. We found that South Korea's results are highly sensitive to the presence of chemical sector in the analysis (Table 2). Value of US decreased but it jumped in 2005-2008 period because of the extraordinary maturity adaptation performance in carbon and graphite electrodes (HS-854519). For MENA countries, the maturity adaptation in Egypt, Kuwait, Lebanon, Qatar, Tunisia, and Yemen diminished. We found that the maturity adaptation decreases in Algeria, Jordan, Morocco, and Saudi Arabia when chemical sector is excluded. Moreover, even though chemical industry in Algeria, Bahrain, Egypt and Saudi Arabia has important weight in total export basket, they could not adaptively manage product development process since their maturity adaptation values including all products are less than the excluding chemical sector for at least three periods. To a lesser extent, we observed similar findings for Germany and US but their total export share of chemical industry is smaller than the MENA. Therefore, this situation does not affect their competitiveness so much.

When we compare the results of for all products with the one excluding rubber and plastic industry for each period, China's maturity adaptation performance in medium-low tech industries showed an improving performance in the model for all products (see Table 3). While adaptation performance of Germany and US is developing in both groups, performance of South Korea turned out to be good in industries based on non-plastic such as building of ships and boats, non-metallic mineral products and basic and fabricated metals. Within MENA group, Egypt, Morocco, Qatar and Saudi Arabia enhanced their adaptation for both product groups over time. Bahrain, Kuwait and Turkey are the countries improving their maturity adaptation in all products.

Findings of low-tech industries, which consist of textile, food and beverage, garment and tobacco, paper and recycling industries, presented in Table 4. The findings show that China, South Korea and US has become more adaptive in low tech industries at end of the period. Although Germany had very high adaptation value, it was not as high as at the beginning. This finding may imply that although these countries - except China- moved production chains to less developed countries, they keep product development stages at home. Adaptation level of the most of MENA countries sharply fell in last period. Substantial developments in benchmark countries leads to the products MENA countries exports get older. Even though most of MENA countries have negative values at extraordinary levels in last period, other countries have declining performance over time as well. Only Egypt in this group showed improvement. 
Table 1: Weighted average results of high-tech products

\begin{tabular}{|c|c|c|c|c|}
\hline \multirow{2}{*}{ Country } & 1996-2000 & 2001-2004 & 2005-2008 & 2009-2013 \\
\hline & all & all & all & all \\
\hline Algeria & -0.31 & 21.28 & 2.28 & -3.33 \\
\hline Arab Emirates & & & -6.59 & \\
\hline Bahrain & & -5.06 & 1.77 & -2.68 \\
\hline China & -0.04 & 1.02 & 10.40 & 3.53 \\
\hline Egypt & 0.30 & -29.60 & 5.33 & 8.10 \\
\hline Germany & -1.49 & -16.38 & 1.17 & 2.64 \\
\hline Iran & & 0.10 & & \\
\hline Jordan & & -1.65 & 0.61 & -1.75 \\
\hline Kuwait & & 43.98 & & 0.49 \\
\hline Lebanon & & -3.28 & -13.55 & -2.55 \\
\hline Morocco & 1.85 & 0.38 & -5.88 & 1.66 \\
\hline Oman & 4.89 & -2.32 & 0.65 & 0.90 \\
\hline Palestine & & & & -10.79 \\
\hline Qatar & & -2.89 & 0.30 & -9.57 \\
\hline Saudi Arabia & 1.34 & -12.60 & -0.45 & 5.71 \\
\hline South Korea & 10.02 & 1.26 & -7.02 & -0.38 \\
\hline Syria & & 0.35 & 1.69 & \\
\hline Tunisia & 0.30 & -0.51 & -7.23 & -0.78 \\
\hline Turkey & 2.67 & 2.18 & -3.83 & -16.10 \\
\hline US & 0.52 & -1.93 & -0.45 & 7.45 \\
\hline Yemen & & & 5.75 & 1.67 \\
\hline
\end{tabular}

Source: Authors' calculations based on COMTRADE (2014)

Table 2: Weighted average results of medium high-tech products

\begin{tabular}{|c|c|c|c|c|c|c|c|c|}
\hline \multirow{2}{*}{ Country } & \multicolumn{2}{|c|}{ 1996-2000 } & \multicolumn{2}{|c|}{ 2001-2004 } & \multicolumn{2}{|c|}{ 2005-2008 } & \multicolumn{2}{|c|}{ 2009-2013 } \\
\hline & all & e.c.i ${ }^{*}$ & all & e.c.i ${ }^{*}$ & all & e.c.i ${ }^{*}$ & all & e.c.i ${ }^{*}$ \\
\hline Algeria & -0.48 & 0.24 & -1.41 & 0.48 & -1.05 & 1.57 & 8.40 & -3.81 \\
\hline Arab Emirates & & & & & -17.21 & -21.13 & & \\
\hline Bahrain & & & 0.04 & 0.19 & 0.45 & 1.19 & 0.30 & 1.87 \\
\hline China & 1.35 & 0.92 & -2.63 & -3.39 & 1.90 & 2.04 & 9.91 & 11.11 \\
\hline Egypt & 0.08 & 3.69 & 0.62 & 2.89 & 1.02 & 0.40 & -2.10 & -1.09 \\
\hline Germany & 4.29 & 6.72 & 1.34 & 1.87 & -3.22 & -2.70 & 1.21 & 1.41 \\
\hline Iran & & & -0.82 & 3.46 & & & & \\
\hline Jordan & & & -1.49 & 1.03 & -1.37 & -4.81 & 2.74 & 0.06 \\
\hline Kuwait & & & 4.20 & 1.67 & & & 0.11 & 0.55 \\
\hline Lebanon & & & 2.56 & 5.35 & -0.54 & -1.13 & -2.62 & -2.80 \\
\hline Morocco & -1.03 & 0.32 & 1.03 & 0.29 & -0.70 & -1.14 & 176.66 & -0.40 \\
\hline Oman & -155.2 & -181.45 & 0.43 & 1.26 & -0.37 & -1.02 & 0.35 & 2.21 \\
\hline Palestine & & & & & & & -7.75 & 1.22 \\
\hline Qatar & & & 6.17 & 0.90 & 68.14 & -3.00 & -0.51 & -1.19 \\
\hline Saudi Arabia & -4.50 & 0.06 & -48.05 & -17.03 & 0.11 & 3.08 & 2.14 & -0.42 \\
\hline South Korea & 1.58 & 0.15 & -3.97 & 0.51 & 0.59 & 0.44 & -0.19 & -6.74 \\
\hline Syria & & & -1.53 & -5.96 & -1.99 & -4.40 & & \\
\hline Tunisia & 23.87 & -4.61 & 5.21 & 8.21 & 3.16 & 3.00 & -7.70 & -9.04 \\
\hline Turkey & 0.51 & -0.53 & -695.08 & -780.68 & 1.39 & 1.86 & -1.45 & 1.36 \\
\hline US & 5.69 & 6.59 & 3.67 & 3.70 & 1886.50 & 2610.90 & 2.54 & 1.18 \\
\hline Yemen & & & & & 3.03 & 2.19 & 1.02 & -0.25 \\
\hline
\end{tabular}

Source: Authors' calculations based on COMTRADE (2014)

* Excluding chemical industry. 
Table 3: Weighted average results of medium low-tech products

\begin{tabular}{|c|c|c|c|c|c|c|c|c|}
\hline \multirow{2}{*}{ Country } & \multicolumn{2}{|c|}{ 1996-2000 } & \multicolumn{2}{|c|}{ 2001-2004 } & \multicolumn{2}{|c|}{ 2005-2008 } & \multicolumn{2}{|c|}{ 2009-2013 } \\
\hline & all & exc. & all & exc. & all & exc. & all & exc. \\
\hline Algeria & 1.28 & 1.22 & 38.16 & 38.32 & 2.10 & 2.49 & -0.47 & -0.49 \\
\hline Arab Emirates & & & & & -3.32 & -2.91 & & \\
\hline Bahrain & & & -9.64 & 0.55 & 1.82 & 1.61 & -1.90 & -2.43 \\
\hline China & -0.81 & -2.29 & 0.59 & -0.57 & 5.96 & 6.09 & 6.41 & -11.36 \\
\hline Egypt & -2.20 & -2.84 & -1.46 & -2.20 & 7.72 & 6.47 & 0.16 & 0.42 \\
\hline Germany & -5.00 & -6.01 & 2.03 & 0.15 & 4.45 & 5.19 & -1.97 & 2.01 \\
\hline Iran & & & -13.78 & 0.09 & & & & \\
\hline Jordan & & & 0.10 & -3.57 & 0.23 & 0.92 & -4.58 & -6.06 \\
\hline Kuwait & & & -36.85 & 2.91 & & & -0.57 & -0.63 \\
\hline Lebanon & & & -5.27 & -5.59 & 4.02 & 4.86 & -6.27 & -11.56 \\
\hline Morocco & 2.34 & 5.07 & 17.86 & 21.85 & -3.60 & -4.91 & 7.12 & 9.13 \\
\hline Oman & 1.16 & 1.72 & -0.62 & -0.08 & -0.68 & -0.36 & -26.23 & -38.89 \\
\hline Palestine & & & & & & & 3.92 & 4.03 \\
\hline Qatar & & & 0.92 & 0.14 & -0.21 & -0.21 & 9.36 & 9.32 \\
\hline Saudi Arabia & -2.14 & -1.87 & -14.88 & -10.10 & 2.27 & 2.66 & -1.12 & 0.16 \\
\hline South Korea & 1.49 & -0.25 & -0.97 & -2.52 & 19.01 & 20.88 & -20.41 & 0.28 \\
\hline Syria & & & 3.08 & -0.03 & -6.25 & -5.29 & & \\
\hline Tunisia & 0.07 & 1.26 & -292.04 & 4.55 & 1.35 & 1.00 & -0.56 & -1.03 \\
\hline Turkey & -0.80 & -0.83 & 2.17 & 2.89 & -1.37 & -2.07 & 0.90 & -4.28 \\
\hline US & -8.13 & -17.13 & 0.59 & 1.21 & 24.89 & 34.63 & 2.22 & -1.92 \\
\hline Yemen & & & & & 265.65 & 428.51 & -3.88 & -4.85 \\
\hline
\end{tabular}

Source: Authors' calculations based on COMTRADE (2014)

* excluding rubber \& plastic industry

Table 4: Weighted average results of low-tech products

\begin{tabular}{|c|c|c|c|c|}
\hline \multirow{2}{*}{ Country } & 1996-2000 & 2001-2004 & 2005-2008 & 2009-2013 \\
\hline & all & all & all & all \\
\hline Algeria & 2.23 & -0.02 & 6.88 & -125.30 \\
\hline Arab Emirates & & & -2.39 & \\
\hline Bahrain & & 0.30 & -3.18 & -4.11 \\
\hline China & 0.91 & 4.96 & 6.06 & 6.46 \\
\hline Egypt & 2.28 & -45.01 & 0.69 & 44.85 \\
\hline Germany & 1814.19 & 5.88 & 8.43 & 754.77 \\
\hline Iran & & 0.71 & & \\
\hline Jordan & & 0.59 & 0.29 & -55.52 \\
\hline Kuwait & & 44.01 & & -189.40 \\
\hline Lebanon & & 23.57 & -2.54 & -894.74 \\
\hline Morocco & 2.76 & -3.22 & 1.23 & -656.87 \\
\hline Oman & -2.81 & 3.64 & 0.08 & -381.61 \\
\hline Palestine & & & & -51.43 \\
\hline Qatar & & 2.11 & 4.44 & -5.54 \\
\hline Saudi Arabia & 1.24 & 12.10 & 2.51 & -109.68 \\
\hline South Korea & 1.58 & 2.19 & -2.17 & 65.38 \\
\hline Syria & & -3.32 & -401.12 & \\
\hline Tunisia & -3.28 & 55.38 & 15.66 & -298.96 \\
\hline Turkey & 14.75 & 6.69 & 2.36 & -11.01 \\
\hline US & -0.13 & 23.93 & 1.39 & 2.17 \\
\hline Yemen & & & -5.63 & -1.54 \\
\hline
\end{tabular}

Source: Authors' calculations based on COMTRADE (2014) 
Finally, we present the 150 products in terms of last five-year average world trade share and with positive maturity growth in Table 5. First of all, the results of benchmark countries did not differ so much in both analyses, implying that their specialization is weighted on nonchemical and non-plastic industries. Secondly, with the similar findings of Germany above, their adaptation increased over time. The results of the performance of MENA countries are sensitive to inclusion of plastic and chemical industries. Inclusion of these two industries into the model inflated dramatically the results in Jordan, Lebanon, Oman, and Saudi Arabia. Lastly, in most of the countries in the group -except Egypt and Oman- maturity adaptation felt dramatically, compared to first period.

Table 5: Weighted average results of 150 products with highest share and positive maturity growth

\begin{tabular}{|c|c|c|c|c|c|c|c|c|}
\hline \multirow[b]{2}{*}{ Country } & \multicolumn{2}{|c|}{ 1996-2000 } & \multicolumn{2}{|c|}{ 2001-2004 } & \multicolumn{2}{|c|}{ 2005-2008 } & \multicolumn{2}{|c|}{ 2009-2013 } \\
\hline & all & $\begin{array}{c}\text { exc. } \\
\text { chemical } \\
\text { \& plastic }\end{array}$ & all & $\begin{array}{c}\text { exc. } \\
\text { chemical } \\
\text { \& plastic }\end{array}$ & all & $\begin{array}{c}\text { exc. } \\
\text { chemical } \\
\text { \& plastic }\end{array}$ & all & $\begin{array}{c}\text { exc. } \\
\text { chemical } \\
\text { \& plastic }\end{array}$ \\
\hline Algeria & -1.22 & -2.03 & 0.56 & 0.01 & -1.74 & -2.39 & -4.20 & -387.95 \\
\hline Arab Emirates & & & & & -20.44 & -22.23 & & \\
\hline Bahrain & & & 0.81 & 0.25 & 9.67 & 9.32 & 0.79 & 0.77 \\
\hline China & 0.41 & 0.26 & 2.82 & 2.77 & 7.71 & 7.68 & 7.68 & 6.49 \\
\hline Egypt & -2.00 & -3.65 & 2.28 & 0.42 & 4.20 & 3.72 & 44.77 & 73.01 \\
\hline Germany & 344.30 & 346.88 & -0.29 & 0.92 & 0.95 & 1.07 & 119.75 & 120.77 \\
\hline Iran & & & -7.56 & 1.77 & & & & \\
\hline Jordan & & & 2.64 & 2.07 & -5.53 & -5.21 & -256.22 & -363.44 \\
\hline Kuwait & & & -1.19 & 2.78 & & & -4.98 & -17.88 \\
\hline Lebanon & & & -20.03 & -13.53 & -5.15 & -4.83 & -768.61 & -848.62 \\
\hline Morocco & 0.65 & 0.86 & 0.37 & -0.13 & -1.22 & -1.20 & -571.86 & -575.29 \\
\hline Oman & -235.80 & -181.93 & 5.44 & 5.35 & -0.73 & -1.21 & -22.33 & -141.81 \\
\hline Palestine & & & & & & & -40.21 & -66.01 \\
\hline Qatar & & & 8.07 & 7.57 & 68.31 & 1.74 & -0.18 & -6.48 \\
\hline Saudi Arabia & -6.30 & 0.99 & -58.30 & -33.33 & 0.73 & 5.53 & -2.83 & -45.53 \\
\hline South Korea & 2.17 & 0.32 & 1.65 & 1.03 & 5.80 & 6.03 & 4.51 & 4.97 \\
\hline Syria & & & 1.38 & -4.64 & -10.60 & -1.65 & & \\
\hline Tunisia & -1.81 & -1.56 & -56.80 & 5.42 & 4.38 & 4.38 & -399.09 & -414.62 \\
\hline Turkey & 1.14 & 0.95 & 2.57 & 2.83 & 2.22 & 1.63 & -16.05 & -17.40 \\
\hline US & 1.15 & 0.50 & -0.18 & 0.96 & 7.21 & 9.12 & 2.11 & 1.90 \\
\hline Yemen & & & & & 7.02 & 8.97 & -3.40 & -2.72 \\
\hline
\end{tabular}

Source: Authors' calculations based on COMTRADE (2014) database

\section{Conclusion}

In this paper, we form a maturity index representing product innovativeness at national and global scale by assuming the existence of significant interrelation between product life cycle and innovative efforts to see the extent to which leading exporting and MENA countries devote their efforts with the direction of global trends. We found that while all benchmark countries improved their adaptation to innovativeness over time in the highest trade share and low-tech products, only China, Germany and US in this group had this performance in hightech and medium-low tech industries, according to weighted average relative maturity change findings. Our results imply that China and, to a lesser extent, US are the best performers in 
medium-high-tech products. When the rubber industry is excluded in medium-low tech, China's adaptation fell, and the others rose.

In MENA group, only Bahrain, Egypt, Lebanon and Saudi Arabia have developed product rejuvenation in high tech industries. Especially, Egypt is among the best performers -including benchmark countries- in last period, not only in high tech, but also in low-tech and highest trade share products. The products of Turkey and Algeria in these categories have fallen even further behind the global market.

In medium-high tech industries, the number of countries that adaptation performance diminished increases when chemical industries are excluded. In most of the periods Algeria, Bahrain, Egypt and Saudi Arabia's chemical industries lowered the weighted averaged adaptation, albeit they specialize in these industries. We obtained the same results for Morocco, Oman and Saudi Arabia, when rubber and plastic products in medium-low tech industries are included.

From this perspective we suggest that MENA countries should diversify their innovative efforts towards high technology intensive and demanded products at the first place and harmonize petroleum derivative products' developments to enjoy high price advantages and able to pay high wages to their workers. Further research on this issue should be on the extension of the analysis by adding other countries and investigating the effect of maturity on productivity which is said to be key source of economic growth and higher living standards.

\section{References}

Abernathy, W. J., \& Utterback, J. M. (1978). Patterns of Industrial Innovation. Technology review, 80(7), 40-47.

Audestsch, D., \& Sanders, M. (2007). Globalization and the rise of the entrepreneurial economy. Jena Economic Research Papers 2007-003.

Audrestsch, D. B., Sanders, M., \& Zhang, L. (2012). How Exports Matter: Trade Patterns Over Development Stages. CEPR.

Audretsch, D. B. (1987). An Empirical Test of the Industry Life Cycle. Weltwirtschaftliches Archiv, 123(2), 297-308. doi:10.1007/BF02706664

Audretsch, D. B., \& Feldman, M. P. (1996). Innovative Clusters and the Industry Life Cycle. Review of Industrial Organization, 11, 253-273. doi:10.1007/BF00157670

Baldwin, R. E. (1971). Determinants of the Commodity Structure of U.S. Trade. The American Economic Review, 61(1), 126-146.

COMTRADE (2014) United Nations Statistics Division. UN COMTRADE. New York :United Nations.

Gardner, D. M. (1986). The Product Life Cycle: It's Role in Marketing Strategy/Some Evolving Observations about the Life Cycle. BEBR Faculty Working Paper No. 1304, Urbana-Champaign. Gruber, W., Mehta, D., \& Vernon, R. (tarih yok). The R \& D Factor in International Trade and International Investment of United States. Journal of Political Economy, 75(1), 20-37.

Grossman, G. M., \& Helpman, E. (1989). Endogenous Product Cycles. NBER Working Paper No. 2913, Cambridge. doi:10.3386/w2913

Hirsch, S., \& Bijaoi, I. (1985). R\&D Intensity and Export Performance: A Micro View. Weltwirtschaftliches Archiv, 121(2), 238-251. doi:10.1007/BF02705822 
Keesing, D. B. (1967). The Impact of Research and Development on United States Trade. Journal of Political Economy, 75(1), 34-48. doi:10.1086/259236

Klepper, S. (1996). Entry, Exit, Growth, and Innovation over the Product Life Cycle. The American Economic Review, 86(3), 562-583.

Krugman, P. (1979). A Model of Innovation, Technology Transfer, and the World Distribution of Income. Journal of Political Economy, 87(2), 253-266. doi: $10.1086 / 260755$

Lee, H.-H., \& Stone, J. A. (1994). Product and Process Innovation in the Product Life Cycle: Estimates for U. S. Manufacturing. Southern Economic Journal, 60(3), 754-763. doi:10.2307/1060581

Levitt, T. (1965). Exploit the Product Life Cycle. Harvard Business Review.

OECD. (1992). Technology and the Economy: The Key Relationships. Paris: OECD.

OECD. (2011). ISIC Rev. 3 Technology Intensity Definition: Classification of manufacturing industries based on $R \& D$ intensities. Paris: OECD.

Sanders, M. (2005). Technology and the Decline of Unskilled Labour Demand: A Theoretical Analysis of the US and European Labour Markets. Cheltenham, UK: Edward Elgar Publishers.

Sanders, M., Bos, J., \& Economidou, C. (2007). R\&D over the Life Cycle. Tjalling C. Koopmans Research Institute Discussion Paper Series 07-18, Utrecht.

Stadler, M. (1991). R\&D Dynamics in the Product Life Cycle. Journal of Evolutionary Economics, 1, 293-305.doi:10.1007/BF01236495

Taymaz, E., Voyvoda, E., \& Y1lmaz, K. (2011). Uluslararası Üretim Zincirlerinde Dönüşüm ve Türkiye'nin Konити. Istanbul: TUSIAD-Koc Universitesi Ekonomik Arastirma Forumu.

Tellis, G. J., \& Crawford, C. M. (1981). An Evolutionary Approach to Product Growth Theory. Journal of Marketing, 45(4), 125-132. doi:10.2307/1251480

United Nations. (2014). UN COMTRADE database. New York: United Nations.

Vernon, R. (1966). International Investment and International Trade in the Product Cycle. The Quarterly Journal of Economics, 80(2), 190-207. doi:10.2307/1880689 


\section{APPENDIX A}

Table 1 Selected 150 products with highest maturity value in 2013

\begin{tabular}{|c|c|c|c|}
\hline $\begin{array}{l}\text { Tech. } \\
\text { Classification }\end{array}$ & Product code & $\begin{array}{c}\text { Average trade share } \\
(2009-2013)\end{array}$ & $\begin{array}{c}\text { Maturity value } \\
(2013)\end{array}$ \\
\hline Low-tech & 610792 & 0.00001 & 3.70 \\
\hline High-tech & 851782 & 0.00323 & 3.03 \\
\hline Low-tech & 482311 & 0.00011 & 2.96 \\
\hline High-tech & 852110 & 0.00138 & 2.30 \\
\hline Medium low-tech & 721149 & 0.00002 & 2.23 \\
\hline Medium low-tech & 721540 & 0.00004 & 2.13 \\
\hline Medium high-tech & 282420 & 0.00000 & 2.04 \\
\hline High-tech & 901020 & 0.00045 & 1.98 \\
\hline Low-tech & 482319 & 0.00001 & 1.92 \\
\hline Medium high-tech & 320643 & 0.00000 & 1.89 \\
\hline High-tech & 846921 & 0.00000 & 1.64 \\
\hline Medium low-tech & 401091 & 0.00007 & 1.51 \\
\hline Medium low-tech & 721339 & 0.00003 & 1.43 \\
\hline Medium high-tech & 283020 & 0.00000 & 1.09 \\
\hline High-tech & 901110 & 0.00014 & 0.94 \\
\hline Medium high-tech & 310270 & 0.00000 & 0.81 \\
\hline High-tech & 293610 & 0.00000 & 0.80 \\
\hline Low-tech & 551433 & 0.00000 & 0.73 \\
\hline High-tech & 910819 & 0.00001 & 0.72 \\
\hline Low-tech & 551529 & 0.00002 & 0.67 \\
\hline Low-tech & 441139 & 0.00000 & 0.65 \\
\hline High-tech & 852090 & 0.00000 & 0.59 \\
\hline Low-tech & 950310 & 0.00000 & 0.59 \\
\hline Low-tech & 430213 & 0.00000 & 0.59 \\
\hline Medium high-tech & 282620 & 0.00000 & 0.58 \\
\hline Medium low-tech & 700410 & 0.00001 & 0.58 \\
\hline Medium high-tech & 854451 & 0.00001 & 0.55 \\
\hline Medium low-tech & 720390 & 0.00003 & 0.53 \\
\hline High-tech & 900912 & 0.00000 & 0.52 \\
\hline Medium high-tech & 845690 & 0.00004 & 0.52 \\
\hline Low-tech & 441229 & 0.00000 & 0.51 \\
\hline Low-tech & 520613 & 0.00000 & 0.51 \\
\hline Low-tech & 520514 & 0.00004 & 0.49 \\
\hline Low-tech & 620819 & 0.00001 & 0.49 \\
\hline Low-tech & 430220 & 0.00000 & 0.49 \\
\hline Medium low-tech & 392072 & 0.00000 & 0.48 \\
\hline Low-tech & 551431 & 0.00000 & 0.48 \\
\hline Low-tech & 540620 & 0.00000 & 0.47 \\
\hline High-tech & 854219 & 0.02121 & 0.47 \\
\hline High-tech & 300331 & 0.00001 & 0.46 \\
\hline Medium low-tech & 700330 & 0.00001 & 0.43 \\
\hline Low-tech & 621310 & 0.00000 & 0.43 \\
\hline High-tech & 847192 & 0.00333 & 0.42 \\
\hline High-tech & 854012 & 0.00000 & 0.42 \\
\hline
\end{tabular}


Table 1 (cont.) Selected 150 products with highest maturity value in 2013

\begin{tabular}{|c|c|c|c|}
\hline $\begin{array}{l}\text { Tech. } \\
\text { Classification }\end{array}$ & Product code & $\begin{array}{c}\text { Average trade share } \\
(2009-2013)\end{array}$ & $\begin{array}{c}\text { Maturity value } \\
\text { (2013) }\end{array}$ \\
\hline Medium high-tech & 845610 & 0.00035 & 0.41 \\
\hline Low-tech & 520631 & 0.00000 & 0.40 \\
\hline Low-tech & 521221 & 0.00000 & 0.39 \\
\hline Medium high-tech & 854310 & 0.00001 & 0.39 \\
\hline Medium high-tech & 280540 & 0.00001 & 0.39 \\
\hline Medium high-tech & 291819 & 0.00006 & 0.38 \\
\hline High-tech & 910820 & 0.00002 & 0.38 \\
\hline High-tech & 900640 & 0.00001 & 0.38 \\
\hline Low-tech & 610321 & 0.00000 & 0.38 \\
\hline Medium high-tech & 291523 & 0.00000 & 0.36 \\
\hline Medium high-tech & 930310 & 0.00000 & 0.35 \\
\hline Medium low-tech & 721020 & 0.00002 & 0.35 \\
\hline High-tech & 911019 & 0.00000 & 0.34 \\
\hline Medium high-tech & 293410 & 0.00013 & 0.34 \\
\hline Medium low-tech & 400591 & 0.00019 & 0.33 \\
\hline Low-tech & 710399 & 0.00016 & 0.33 \\
\hline Medium high-tech & 282734 & 0.00000 & 0.33 \\
\hline Medium low-tech & 721129 & 0.00007 & 0.32 \\
\hline Low-tech & 540342 & 0.00000 & 0.32 \\
\hline Low-tech & 521112 & 0.00000 & 0.32 \\
\hline Low-tech & 950330 & 0.00000 & 0.32 \\
\hline High-tech & 900792 & 0.00001 & 0.30 \\
\hline High-tech & 847340 & 0.00026 & 0.30 \\
\hline Low-tech & 551120 & 0.00001 & 0.30 \\
\hline High-tech & 910899 & 0.00000 & 0.30 \\
\hline Low-tech & 950320 & 0.00000 & 0.30 \\
\hline High-tech & 293910 & 0.00005 & 0.30 \\
\hline High-tech & 293319 & 0.00009 & 0.29 \\
\hline Low-tech & 540610 & 0.00001 & 0.29 \\
\hline High-tech & 852210 & 0.00000 & 0.29 \\
\hline Medium high-tech & 844400 & 0.00009 & 0.29 \\
\hline Low-tech & 630641 & 0.00000 & 0.28 \\
\hline Low-tech & 580133 & 0.00001 & 0.28 \\
\hline Medium low-tech & 810192 & 0.00000 & 0.28 \\
\hline Low-tech & 950341 & 0.00000 & 0.28 \\
\hline Medium low-tech & 722870 & 0.00006 & 0.28 \\
\hline Medium high-tech & 293379 & 0.00032 & 0.28 \\
\hline Medium high-tech & 283326 & 0.00000 & 0.27 \\
\hline Medium high-tech & 291469 & 0.00002 & 0.27 \\
\hline Low-tech & 570252 & 0.00000 & 0.27 \\
\hline Low-tech & 520512 & 0.00021 & 0.27 \\
\hline Low-tech & 610453 & 0.00004 & 0.26 \\
\hline Low-tech & 551634 & 0.00000 & 0.26 \\
\hline Low-tech & 551322 & 0.00000 & 0.25 \\
\hline Low-tech & 610290 & 0.00001 & 0.25 \\
\hline High-tech & 852820 & 0.00002 & 0.25 \\
\hline
\end{tabular}


Table 1 (cont.) Selected 150 products with highest maturity value in 2013

\begin{tabular}{|c|c|c|c|}
\hline $\begin{array}{l}\text { Tech. } \\
\text { Classification }\end{array}$ & Product code & $\begin{array}{c}\text { Average trade share } \\
(2009-2013)\end{array}$ & $\begin{array}{c}\text { Maturity value } \\
(2013)\end{array}$ \\
\hline Low-tech & 610459 & 0.00002 & 0.25 \\
\hline Medium high-tech & 330125 & 0.00002 & 0.25 \\
\hline Medium high-tech & 152010 & 0.00003 & 0.24 \\
\hline Low-tech & 610333 & 0.00002 & 0.24 \\
\hline Low-tech & 530620 & 0.00001 & 0.24 \\
\hline Medium high-tech & 280120 & 0.00008 & 0.24 \\
\hline Medium low-tech & 681591 & 0.00002 & 0.24 \\
\hline Medium high-tech & 840611 & 0.00001 & 0.24 \\
\hline Low-tech & 950349 & 0.00000 & 0.24 \\
\hline Low-tech & 520611 & 0.00001 & 0.24 \\
\hline Low-tech & 621430 & 0.00007 & 0.24 \\
\hline Low-tech & 620640 & 0.00033 & 0.24 \\
\hline Medium high-tech & 291430 & 0.00002 & 0.24 \\
\hline Low-tech & 510910 & 0.00001 & 0.24 \\
\hline Low-tech & 710229 & 0.00003 & 0.23 \\
\hline Low-tech & 510510 & 0.00000 & 0.23 \\
\hline Medium low-tech & 720291 & 0.00002 & 0.23 \\
\hline Medium high-tech & 843352 & 0.00001 & 0.22 \\
\hline Low-tech & 580430 & 0.00000 & 0.22 \\
\hline Low-tech & 550969 & 0.00002 & 0.22 \\
\hline Medium high-tech & 710420 & 0.00001 & 0.22 \\
\hline Low-tech & 710490 & 0.00004 & 0.22 \\
\hline Low-tech & 520622 & 0.00001 & 0.21 \\
\hline Low-tech & 530710 & 0.00002 & 0.21 \\
\hline Low-tech & 520535 & 0.00000 & 0.21 \\
\hline Low-tech & 551614 & 0.00001 & 0.21 \\
\hline High-tech & 300339 & 0.00004 & 0.21 \\
\hline Low-tech & 520922 & 0.00001 & 0.21 \\
\hline Low-tech & 610439 & 0.00002 & 0.21 \\
\hline Low-tech & 610469 & 0.00005 & 0.21 \\
\hline Medium high-tech & 310229 & 0.00002 & 0.20 \\
\hline Low-tech & 570251 & 0.00000 & 0.20 \\
\hline High-tech & 847290 & 0.00043 & 0.20 \\
\hline Medium high-tech & 842832 & 0.00003 & 0.20 \\
\hline Medium high-tech & 290243 & 0.00104 & 0.20 \\
\hline Low-tech & 610433 & 0.00004 & 0.20 \\
\hline Low-tech & 540824 & 0.00000 & 0.20 \\
\hline High-tech & 900311 & 0.00012 & 0.20 \\
\hline Low-tech & 710391 & 0.00018 & 0.20 \\
\hline High-tech & 910212 & 0.00009 & 0.20 \\
\hline Medium high-tech & 330114 & 0.00000 & 0.20 \\
\hline Medium high-tech & 870130 & 0.00008 & 0.20 \\
\hline Medium high-tech & 290314 & 0.00000 & 0.20 \\
\hline High-tech & 847191 & 0.00371 & 0.20 \\
\hline Medium high-tech & 291812 & 0.00002 & 0.20 \\
\hline High-tech & 293430 & 0.00001 & 0.20 \\
\hline
\end{tabular}


Table 1 (cont.) Selected 150 products with highest maturity value in 2013

\begin{tabular}{lccc}
\hline $\begin{array}{l}\text { Tech. } \\
\text { Classification }\end{array}$ & Product code & $\begin{array}{c}\text { Average trade share } \\
(\mathbf{2 0 0 9 - 2 0 1 3 )}\end{array}$ & $\begin{array}{c}\text { Maturity value } \\
(\mathbf{2 0 1 3})\end{array}$ \\
\hline Low-tech & 630293 & 0.00002 & 0.20 \\
High-tech & 910811 & 0.00008 & 0.20 \\
Low-tech & 520621 & 0.00000 & 0.20 \\
Low-tech & 521119 & 0.00001 & 0.20 \\
Low-tech & 580429 & 0.00002 & 0.20 \\
Low-tech & 530610 & 0.00002 & 0.20 \\
High-tech & 902710 & 0.00026 & 0.19 \\
Medium high-tech & 350290 & 0.00006 & 0.19 \\
Low-tech & 610463 & 0.00020 & 0.19 \\
Low-tech & 521129 & 0.00000 & 0.19 \\
High-tech & 900699 & 0.00004 & 0.19 \\
Low-tech & 510810 & 0.00001 & 0.19 \\
Low-tech & 650400 & 0.00002 & 0.18 \\
High-tech & 293721 & 0.00003 & 0.18 \\
\hline & Total & $\mathbf{0 . 0 3 9 9 7}$ &
\end{tabular}

Source: Authors' calculation based on COMTRADE (2015) database 
Table 2 Selected 150 products with highest maturity growth rate (2009-2013)

\begin{tabular}{|c|c|c|c|}
\hline $\begin{array}{l}\text { Tech. } \\
\text { Classification }\end{array}$ & Product code & $\begin{array}{c}\text { Average trade share } \\
\quad(2009-2013)\end{array}$ & $\begin{array}{c}\text { Maturity growth rate } \\
2009-2013)\end{array}$ \\
\hline Medium high-tech & 854380 & 0.00185 & 4000.16 \\
\hline High-tech & 910812 & 0.00000 & 2240.70 \\
\hline Medium low-tech & 392119 & 0.00020 & 1184.08 \\
\hline High-tech & 902990 & 0.00010 & 220.12 \\
\hline Medium high-tech & 731511 & 0.00011 & 191.60 \\
\hline Low-tech & 530810 & 0.00000 & 172.83 \\
\hline Medium high-tech & 850131 & 0.00048 & 171.05 \\
\hline Medium low-tech & 401695 & 0.00002 & 158.31 \\
\hline High-tech & 901490 & 0.00012 & 146.02 \\
\hline Low-tech & 430211 & 0.00010 & 130.49 \\
\hline High-tech & 911019 & 0.00000 & 121.11 \\
\hline Medium low-tech & 701820 & 0.00001 & 110.82 \\
\hline Low-tech & 630222 & 0.00006 & 106.72 \\
\hline Medium high-tech & 850519 & 0.00012 & 76.51 \\
\hline Medium high-tech & 284011 & 0.00001 & 71.47 \\
\hline Low-tech & 551120 & 0.00001 & 68.45 \\
\hline Medium high-tech & 841360 & 0.00041 & 59.09 \\
\hline Low-tech & 600192 & 0.00007 & 56.70 \\
\hline Medium high-tech & 870893 & 0.00058 & 53.22 \\
\hline Medium low-tech & 401290 & 0.00009 & 48.92 \\
\hline Low-tech & 591140 & 0.00002 & 47.84 \\
\hline Medium high-tech & 841459 & 0.00064 & 47.54 \\
\hline High-tech & 910899 & 0.00000 & 47.11 \\
\hline Medium high-tech & 290361 & 0.00002 & 42.85 \\
\hline High-tech & 902730 & 0.00024 & 42.72 \\
\hline Medium high-tech & 845380 & 0.00001 & 41.16 \\
\hline High-tech & 902219 & 0.00015 & 38.97 \\
\hline Medium low-tech & 720390 & 0.00003 & 38.75 \\
\hline Medium high-tech & 291890 & 0.00013 & 36.83 \\
\hline Medium low-tech & 401693 & 0.00079 & 34.93 \\
\hline Medium high-tech & 290517 & 0.00003 & 34.09 \\
\hline Medium high-tech & 391220 & 0.00003 & 33.29 \\
\hline Low-tech & 630140 & 0.00014 & 33.26 \\
\hline Low-tech & 540262 & 0.00002 & 33.26 \\
\hline Medium high-tech & 381210 & 0.00005 & 32.83 \\
\hline Medium high-tech & 842320 & 0.00001 & 32.49 \\
\hline High-tech & 911490 & 0.00013 & 31.77 \\
\hline Medium high-tech & 390469 & 0.00012 & 30.02 \\
\hline Medium high-tech & 321519 & 0.00045 & 29.74 \\
\hline Medium high-tech & 848390 & 0.00073 & 29.26 \\
\hline High-tech & 294140 & 0.00001 & 29.18 \\
\hline High-tech & 901841 & 0.00004 & 28.88 \\
\hline Medium high-tech & 282510 & 0.00002 & 28.78 \\
\hline High-tech & 293625 & 0.00001 & 27.64 \\
\hline High-tech & 900580 & 0.00003 & 27.55 \\
\hline Medium high-tech & 540231 & 0.00007 & 27.53 \\
\hline
\end{tabular}


Table 2 (cont.) Selected 150 products with highest maturity growth rate (2009-2013)

\begin{tabular}{|c|c|c|c|}
\hline $\begin{array}{l}\text { Tech. } \\
\text { Classification }\end{array}$ & Product code & $\begin{array}{l}\text { Average trade share } \\
(2009-2013)\end{array}$ & $\begin{array}{l}\text { Maturity growth rate } \\
(2009-2013)\end{array}$ \\
\hline Low-tech & 650590 & 0.00031 & 26.34 \\
\hline Medium high-tech & 854430 & 0.00206 & 26.20 \\
\hline Low-tech & 520611 & 0.00001 & 25.98 \\
\hline Low-tech & 540772 & 0.00013 & 24.68 \\
\hline Medium low-tech & 392190 & 0.00089 & 23.71 \\
\hline Low-tech & 610459 & 0.00002 & 23.69 \\
\hline Medium low-tech & 401699 & 0.00077 & 23.27 \\
\hline Low-tech & 610899 & 0.00000 & 22.34 \\
\hline Medium high-tech & 844820 & 0.00003 & 22.08 \\
\hline Low-tech & 590320 & 0.00025 & 21.78 \\
\hline Medium high-tech & 280540 & 0.00001 & 21.70 \\
\hline Low-tech & 710391 & 0.00018 & 21.07 \\
\hline Medium high-tech & 848190 & 0.00112 & 21.00 \\
\hline High-tech & 910819 & 0.00001 & 20.97 \\
\hline Low-tech & 610332 & 0.00002 & 20.12 \\
\hline Medium low-tech & 391690 & 0.00013 & 20.07 \\
\hline Medium high-tech & 841939 & 0.00014 & 19.73 \\
\hline Medium high-tech & 842860 & 0.00002 & 18.56 \\
\hline Medium high-tech & 841490 & 0.00110 & 18.30 \\
\hline Low-tech & 590390 & 0.00023 & 18.30 \\
\hline Low-tech & 620520 & 0.00087 & 17.98 \\
\hline Medium high-tech & 848071 & 0.00067 & 17.86 \\
\hline Medium low-tech & 391740 & 0.00035 & 17.70 \\
\hline High-tech & 901410 & 0.00003 & 17.27 \\
\hline Low-tech & 621210 & 0.00065 & 17.12 \\
\hline Low-tech & 420291 & 0.00017 & 16.92 \\
\hline Medium high-tech & 291221 & 0.00001 & 16.83 \\
\hline Low-tech & 520631 & 0.00000 & 16.56 \\
\hline Medium low-tech & 722920 & 0.00004 & 16.54 \\
\hline Medium low-tech & 590610 & 0.00004 & 16.37 \\
\hline Medium low-tech & 391610 & 0.00002 & 15.98 \\
\hline Medium low-tech & 741532 & 0.00003 & 15.73 \\
\hline High-tech & 903120 & 0.00009 & 15.56 \\
\hline Medium high-tech & 842832 & 0.00003 & 14.92 \\
\hline Medium high-tech & 290920 & 0.00000 & 14.83 \\
\hline Medium high-tech & 291531 & 0.00009 & 14.70 \\
\hline Medium high-tech & 310229 & 0.00002 & 14.60 \\
\hline Medium high-tech & 843031 & 0.00009 & 14.17 \\
\hline Medium high-tech & 848240 & 0.00009 & 14.09 \\
\hline Low-tech & 481320 & 0.00006 & 13.76 \\
\hline Medium low-tech & 680100 & 0.00008 & 13.46 \\
\hline Medium high-tech & 843340 & 0.00008 & 13.42 \\
\hline Low-tech & 600230 & 0.00037 & 13.38 \\
\hline Medium high-tech & 310260 & 0.00003 & 13.30 \\
\hline Medium high-tech & 710420 & 0.00001 & 13.17 \\
\hline Medium low-tech & 761100 & 0.00001 & 12.92 \\
\hline
\end{tabular}


Table 2 (cont.) Selected 150 products with highest maturity growth rate (2009-2013)

\begin{tabular}{|c|c|c|c|}
\hline $\begin{array}{l}\text { Tech. } \\
\text { Classification }\end{array}$ & Product code & $\begin{array}{c}\text { Average trade share } \\
(2009-2013)\end{array}$ & $\begin{array}{c}\text { Maturity growth rate } \\
(2009-2013)\end{array}$ \\
\hline High-tech & 901790 & 0.00002 & 12.80 \\
\hline Medium low-tech & 700910 & 0.00021 & 12.76 \\
\hline Medium low-tech & 680423 & 0.00001 & 12.71 \\
\hline High-tech & 300340 & 0.00001 & 12.64 \\
\hline High-tech & 880520 & 0.00008 & 12.59 \\
\hline Medium high-tech & 842290 & 0.00041 & 12.44 \\
\hline Low-tech & 950800 & 0.00007 & 12.43 \\
\hline Medium high-tech & 842430 & 0.00020 & 12.15 \\
\hline Low-tech & 580133 & 0.00001 & 12.14 \\
\hline High-tech & 291821 & 0.00001 & 11.90 \\
\hline Medium low-tech & 392113 & 0.00024 & 11.75 \\
\hline Medium low-tech & 681120 & 0.00004 & 11.69 \\
\hline Medium high-tech & 282759 & 0.00002 & 11.59 \\
\hline Low-tech & 620640 & 0.00033 & 11.56 \\
\hline Medium high-tech & 853921 & 0.00019 & 11.33 \\
\hline Low-tech & 521119 & 0.00001 & 11.20 \\
\hline Low-tech & 940490 & 0.00057 & 11.01 \\
\hline Medium low-tech & 820711 & 0.00004 & 10.92 \\
\hline Low-tech & 551634 & 0.00000 & 10.85 \\
\hline Medium high-tech & 290129 & 0.00019 & 10.80 \\
\hline Medium high-tech & 380991 & 0.00018 & 10.79 \\
\hline Medium high-tech & 290122 & 0.00059 & 10.70 \\
\hline Medium low-tech & 701339 & 0.00012 & 10.46 \\
\hline Low-tech & 531090 & 0.00000 & 10.33 \\
\hline Low-tech & 520613 & 0.00000 & 10.27 \\
\hline Medium high-tech & 843880 & 0.00015 & 10.25 \\
\hline Low-tech & 610453 & 0.00004 & 10.18 \\
\hline Low-tech & 420211 & 0.00007 & 10.04 \\
\hline Low-tech & 540791 & 0.00001 & 9.95 \\
\hline Medium low-tech & 820420 & 0.00007 & 9.84 \\
\hline Low-tech & 620331 & 0.00012 & 9.81 \\
\hline Medium high-tech & 390220 & 0.00006 & 9.76 \\
\hline Medium high-tech & 292142 & 0.00003 & 9.67 \\
\hline Medium high-tech & 390210 & 0.00185 & 9.59 \\
\hline High-tech & 854129 & 0.00098 & 9.58 \\
\hline Medium low-tech & 400821 & 0.00014 & 9.50 \\
\hline Low-tech & 520513 & 0.00004 & 9.48 \\
\hline Low-tech & 611219 & 0.00000 & 9.45 \\
\hline High-tech & 293321 & 0.00001 & 9.42 \\
\hline High-tech & 300331 & 0.00001 & 9.20 \\
\hline Medium high-tech & 732183 & 0.00009 & 9.14 \\
\hline Low-tech & 620343 & 0.00039 & 9.08 \\
\hline High-tech & 910119 & 0.00004 & 9.04 \\
\hline Low-tech & 551614 & 0.00001 & 8.97 \\
\hline Medium low-tech & 401120 & 0.00160 & 8.96 \\
\hline Medium high-tech & 291430 & 0.00002 & 8.89 \\
\hline
\end{tabular}


Table 2 (cont.) Selected 150 products with highest maturity growth rate (2009-2013)

\begin{tabular}{lccc}
\hline $\begin{array}{l}\text { Tech. } \\
\text { Classification }\end{array}$ & Product code & $\begin{array}{c}\text { Average trade share } \\
(\mathbf{2 0 0 9 - 2 0 1 3 )}\end{array}$ & $\begin{array}{c}\text { Maturity growth rate } \\
(\mathbf{2 0 0 9 - 2 0 1 3 )}\end{array}$ \\
\hline Medium low-tech & 731520 & 0.00002 & 8.88 \\
Medium high-tech & 860691 & 0.00003 & 8.86 \\
Medium low-tech & 890200 & 0.00005 & 8.86 \\
Low-tech & 650200 & 0.00000 & 8.80 \\
Low-tech & 650100 & 0.00000 & 8.66 \\
Medium low-tech & 400940 & 0.00004 & 8.56 \\
Medium high-tech & 848490 & 0.00013 & 8.31 \\
Medium high-tech & 390120 & 0.00188 & 8.25 \\
Medium high-tech & 291620 & 0.00004 & 8.20 \\
Low-tech & 410520 & 0.00009 & 8.13 \\
Medium low-tech & 401010 & 0.00009 & 8.13 \\
Low-tech & 560290 & 0.00002 & 8.07 \\
\hline
\end{tabular}

Source: Authors' calculation based on COMTRADE (2015) database 
Table 3 Selected 150 products with highest share and positive maturity growth

\begin{tabular}{|c|c|c|c|}
\hline $\begin{array}{l}\text { Tech. } \\
\text { Classification }\end{array}$ & Product code & $\begin{array}{l}\text { Average trade share } \\
(2009-2013)\end{array}$ & $\begin{array}{l}\text { Maturity growth rate } \\
(2009-2013)\end{array}$ \\
\hline Medium high-tech & 854380 & 0.00185 & 4000.16 \\
\hline Medium high-tech & 870893 & 0.00058 & 53.22 \\
\hline Medium high-tech & 841459 & 0.00064 & 47.54 \\
\hline Medium low-tech & 401693 & 0.00079 & 34.93 \\
\hline Medium high-tech & 848390 & 0.00073 & 29.26 \\
\hline Medium high-tech & 854430 & 0.00206 & 26.20 \\
\hline Medium low-tech & 392190 & 0.00089 & 23.71 \\
\hline Medium low-tech & 401699 & 0.00077 & 23.27 \\
\hline Medium high-tech & 848190 & 0.00112 & 21.00 \\
\hline Medium high-tech & 841490 & 0.00110 & 18.30 \\
\hline Low-tech & 620520 & 0.00087 & 17.98 \\
\hline Medium high-tech & 848071 & 0.00067 & 17.86 \\
\hline Low-tech & 621210 & 0.00065 & 17.12 \\
\hline Low-tech & 940490 & 0.00057 & 11.01 \\
\hline Medium high-tech & 290122 & 0.00059 & 10.70 \\
\hline Medium high-tech & 390210 & 0.00185 & 9.59 \\
\hline High-tech & 854129 & 0.00098 & 9.58 \\
\hline Medium low-tech & 401120 & 0.00160 & 8.96 \\
\hline Medium high-tech & 390120 & 0.00188 & 8.25 \\
\hline Medium low-tech & 392321 & 0.00068 & 7.59 \\
\hline High-tech & 847193 & 0.00614 & 7.08 \\
\hline High-tech & 903289 & 0.00162 & 6.51 \\
\hline Medium low-tech & 392310 & 0.00070 & 6.50 \\
\hline High-tech & 851830 & 0.00058 & 6.45 \\
\hline Medium high-tech & 390799 & 0.00053 & 5.78 \\
\hline High-tech & 851782 & 0.00323 & 5.51 \\
\hline High-tech & 852520 & 0.01360 & 5.41 \\
\hline Medium high-tech & 330210 & 0.00092 & 4.92 \\
\hline Medium high-tech & 290531 & 0.00098 & 4.73 \\
\hline Medium high-tech & 320610 & 0.00065 & 4.65 \\
\hline High-tech & 910211 & 0.00070 & 4.60 \\
\hline Medium high-tech & 290243 & 0.00104 & 4.54 \\
\hline High-tech & 852110 & 0.00138 & 4.48 \\
\hline High-tech & 854219 & 0.02121 & 4.42 \\
\hline Medium high-tech & 390690 & 0.00093 & 4.41 \\
\hline Medium high-tech & 290919 & 0.00054 & 4.39 \\
\hline Medium high-tech & 940540 & 0.00079 & 4.29 \\
\hline Medium high-tech & 940510 & 0.00079 & 4.22 \\
\hline Medium high-tech & 851220 & 0.00084 & 4.13 \\
\hline Medium high-tech & 390720 & 0.00077 & 4.08 \\
\hline High-tech & 903180 & 0.00127 & 4.03 \\
\hline High-tech & 847191 & 0.00371 & 3.97 \\
\hline High-tech & 853400 & 0.00323 & 3.67 \\
\hline Medium high-tech & 870321 & 0.00098 & 3.59 \\
\hline High-tech & 847192 & 0.00333 & 3.25 \\
\hline Medium high-tech & 841391 & 0.00111 & 3.16 \\
\hline
\end{tabular}


Table 3 (cont.) Selected 150 products with highest share and positive maturity growth

\begin{tabular}{|c|c|c|c|}
\hline $\begin{array}{l}\text { Tech. } \\
\text { Classification }\end{array}$ & Product code & $\begin{array}{c}\text { Average trade share } \\
(2009-2013)\end{array}$ & $\begin{array}{c}\text { Maturity growth rate } \\
(2009-2013)\end{array}$ \\
\hline Medium high-tech & 870829 & 0.00428 & 3.06 \\
\hline Medium high-tech & 290250 & 0.00105 & 2.90 \\
\hline Medium high-tech & 841430 & 0.00091 & 2.87 \\
\hline High-tech & 901390 & 0.00062 & 2.85 \\
\hline Low-tech & 940190 & 0.00177 & 2.80 \\
\hline Medium high-tech & 390760 & 0.00088 & 2.79 \\
\hline Medium high-tech & 845710 & 0.00068 & 2.75 \\
\hline Medium high-tech & 850110 & 0.00090 & 2.71 \\
\hline Medium high-tech & 390230 & 0.00088 & 2.56 \\
\hline Medium high-tech & 840734 & 0.00226 & 2.48 \\
\hline Medium high-tech & 390110 & 0.00192 & 2.31 \\
\hline Medium high-tech & 390810 & 0.00081 & 2.29 \\
\hline Medium low-tech & 392010 & 0.00093 & 2.22 \\
\hline Medium high-tech & 390190 & 0.00100 & 2.12 \\
\hline High-tech & 854110 & 0.00077 & 2.08 \\
\hline Medium high-tech & 870431 & 0.00180 & 2.04 \\
\hline Medium low-tech & 392690 & 0.00351 & 2.03 \\
\hline Medium high-tech & 850450 & 0.00072 & 1.99 \\
\hline Medium high-tech & 853690 & 0.00261 & 1.97 \\
\hline High-tech & 852721 & 0.00063 & 1.93 \\
\hline Medium high-tech & 281410 & 0.00065 & 1.91 \\
\hline Medium high-tech & 870323 & 0.01658 & 1.90 \\
\hline Low-tech & 611030 & 0.00142 & 1.87 \\
\hline High-tech & 901839 & 0.00142 & 1.82 \\
\hline Medium high-tech & 870870 & 0.00112 & 1.81 \\
\hline Medium high-tech & 842890 & 0.00061 & 1.78 \\
\hline High-tech & 841112 & 0.00175 & 1.75 \\
\hline Medium high-tech & 841330 & 0.00086 & 1.74 \\
\hline Medium high-tech & 848120 & 0.00058 & 1.71 \\
\hline Medium high-tech & 290511 & 0.00066 & 1.71 \\
\hline High-tech & 851740 & 0.00071 & 1.68 \\
\hline Low-tech & 940320 & 0.00081 & 1.67 \\
\hline Medium high-tech & 870190 & 0.00137 & 1.64 \\
\hline Medium high-tech & 853710 & 0.00256 & 1.61 \\
\hline Medium high-tech & 390740 & 0.00078 & 1.61 \\
\hline Medium low-tech & 731815 & 0.00117 & 1.56 \\
\hline Medium high-tech & 290121 & 0.00053 & 1.49 \\
\hline High-tech & 853224 & 0.00083 & 1.48 \\
\hline High-tech & 880240 & 0.00642 & 1.46 \\
\hline Low-tech & 410431 & 0.00069 & 1.45 \\
\hline Medium high-tech & 840991 & 0.00222 & 1.43 \\
\hline Medium high-tech & 848210 & 0.00094 & 1.42 \\
\hline Medium high-tech & 870899 & 0.00629 & 1.42 \\
\hline Medium high-tech & 841590 & 0.00099 & 1.41 \\
\hline Medium high-tech & 840820 & 0.00210 & 1.41 \\
\hline
\end{tabular}


Table 3 (cont.) Selected 150 products with highest share and positive maturity growth

\begin{tabular}{|c|c|c|c|}
\hline $\begin{array}{l}\text { Tech. } \\
\text { Classification }\end{array}$ & Product code & $\begin{array}{c}\text { Average trade share } \\
(2009-2013)\end{array}$ & $\begin{array}{c}\text { Maturity growth rate } \\
(2009-2013)\end{array}$ \\
\hline Low-tech & 610990 & 0.00067 & 1.38 \\
\hline Medium high-tech & 870410 & 0.00070 & 1.35 \\
\hline Medium high-tech & 870332 & 0.00876 & 1.27 \\
\hline Low-tech & 710239 & 0.00534 & 1.24 \\
\hline Medium high-tech & 870120 & 0.00155 & 1.20 \\
\hline Medium high-tech & 870324 & 0.00824 & 1.17 \\
\hline Medium low-tech & 761690 & 0.00086 & 1.16 \\
\hline Medium high-tech & 870421 & 0.00310 & 1.13 \\
\hline Medium high-tech & 842951 & 0.00075 & 1.13 \\
\hline Low-tech & 620462 & 0.00155 & 1.07 \\
\hline Medium low-tech & 391990 & 0.00084 & 1.06 \\
\hline Medium high-tech & 870422 & 0.00126 & 1.05 \\
\hline High-tech & 910221 & 0.00053 & 1.04 \\
\hline High-tech & 854290 & 0.00222 & 1.04 \\
\hline Medium high-tech & 841810 & 0.00083 & 1.01 \\
\hline Medium high-tech & 842952 & 0.00137 & 0.99 \\
\hline Low-tech & 560300 & 0.00087 & 0.97 \\
\hline Medium low-tech & 690890 & 0.00073 & 0.93 \\
\hline Medium high-tech & 840999 & 0.00227 & 0.90 \\
\hline Low-tech & 940350 & 0.00062 & 0.90 \\
\hline High-tech & 847330 & 0.00962 & 0.89 \\
\hline Low-tech & 940161 & 0.00097 & 0.84 \\
\hline Medium high-tech & 870423 & 0.00099 & 0.80 \\
\hline Medium high-tech & 870333 & 0.00229 & 0.80 \\
\hline Medium low-tech & 721049 & 0.00151 & 0.79 \\
\hline Medium low-tech & 720923 & 0.00058 & 0.78 \\
\hline High-tech & 300439 & 0.00148 & 0.76 \\
\hline Medium high-tech & 841510 & 0.00079 & 0.75 \\
\hline Low-tech & 420221 & 0.00062 & 0.75 \\
\hline Low-tech & 420292 & 0.00102 & 0.70 \\
\hline Low-tech & 640391 & 0.00094 & 0.64 \\
\hline Medium high-tech & 853890 & 0.00201 & 0.64 \\
\hline Low-tech & 620342 & 0.00170 & 0.61 \\
\hline Medium high-tech & 870840 & 0.00366 & 0.61 \\
\hline Medium high-tech & 840890 & 0.00086 & 0.61 \\
\hline Medium high-tech & 843149 & 0.00180 & 0.59 \\
\hline Medium high-tech & 853120 & 0.00071 & 0.59 \\
\hline Medium low-tech & 720824 & 0.00142 & 0.59 \\
\hline Low-tech & 640419 & 0.00076 & 0.55 \\
\hline Medium high-tech & 390410 & 0.00077 & 0.51 \\
\hline High-tech & 902780 & 0.00076 & 0.35 \\
\hline Low-tech & 940360 & 0.00161 & 0.32 \\
\hline Medium low-tech & 721331 & 0.00069 & 0.30 \\
\hline High-tech & 901380 & 0.00476 & 0.29 \\
\hline Medium high-tech & 293339 & 0.00093 & 0.28 \\
\hline Medium low-tech & 392410 & 0.00059 & 0.28 \\
\hline
\end{tabular}


Table 3 (cont.) Selected 150 products with highest share and positive maturity growth

\begin{tabular}{lccc}
\hline $\begin{array}{l}\text { Tech. } \\
\text { Classification }\end{array}$ & Product code & $\begin{array}{c}\text { Average trade share } \\
(\mathbf{2 0 0 9 - 2 0 1 3 )}\end{array}$ & $\begin{array}{c}\text { Maturity growth rate } \\
(\mathbf{2 0 0 9 - 2 0 1 3 )}\end{array}$ \\
\hline Medium low-tech & 720712 & 0.00101 & 0.27 \\
Medium high-tech & 870331 & 0.00142 & 0.26 \\
Medium high-tech & 380820 & 0.00057 & 0.26 \\
High-tech & 293799 & 0.00062 & 0.25 \\
High-tech & 293359 & 0.00119 & 0.21 \\
Low-tech & 480100 & 0.00065 & 0.19 \\
High-tech & 851710 & 0.00061 & 0.19 \\
Low-tech & 481011 & 0.00093 & 0.17 \\
Medium low-tech & 720823 & 0.00063 & 0.09 \\
Medium high-tech & 380830 & 0.00076 & 0.07 \\
High-tech & 300431 & 0.00068 & 0.02 \\
Low-tech & 481840 & 0.00091 & 0.00 \\
Low-tech & 640399 & 0.00214 & 0.00 \\
\hline
\end{tabular}

Source: Authors' calculation based on COMTRADE (2015) database 


\section{APPENDIX B}

Figure 1: Maturity index values of electrical machines and apparatus, nes (854380) at national and global level, 1996-2013

\begin{tabular}{|c|c|c|c|c|c|c|c|c|c|c|c|c|c|c|}
\hline Year & World & Algeria & Bahrain & China & Egypt & Germany & Jordan & Lebanon & Morocco & S. Arabia & S. Korea & Tunisia & Turkey & USA \\
\hline 1996 & 0.031 & 0.522 & & 0.039 & & 0.129 & & & 0.402 & 0.322 & -0.232 & 0.314 & 0.047 & -0.124 \\
\hline 1997 & 0.018 & -1.011 & & 0.039 & -2.692 & 0.079 & & -0.233 & 0.488 & & -0.291 & -0.011 & 0.339 & -0.060 \\
\hline 1998 & -0.009 & & & 0.038 & -1.720 & 0.050 & & -0.246 & 0.429 & -0.304 & -0.288 & 0.214 & 0.452 & 0.003 \\
\hline 1999 & -0.033 & 0.113 & & 0.030 & -0.768 & 0.031 & 0.072 & -0.170 & 0.384 & -0.131 & -0.080 & 0.700 & 0.503 & 0.098 \\
\hline 2000 & -0.067 & -0.075 & & 0.043 & 0.291 & 0.013 & -0.160 & 0.065 & 0.339 & 0.119 & 0.031 & 1.175 & 0.593 & 0.159 \\
\hline 2001 & -0.066 & -0.333 & & 0.037 & 1.036 & 0.006 & -0.287 & 0.110 & 0.310 & 0.425 & 0.211 & 1.399 & 0.471 & 0.186 \\
\hline 2002 & -0.076 & -0.483 & & 0.039 & 1.524 & 0.011 & -0.411 & 0.244 & 0.487 & -0.455 & 0.191 & 1.443 & 0.417 & 0.158 \\
\hline 2003 & -0.109 & -0.849 & & 0.064 & 2.039 & 0.008 & -0.555 & 0.308 & 0.494 & -0.022 & 0.086 & 1.162 & 0.399 & 0.095 \\
\hline 2004 & -0.136 & -0.961 & & 0.105 & 3.235 & 0.005 & -0.632 & 0.369 & 0.429 & 0.349 & -0.019 & 0.843 & 0.375 & 0.035 \\
\hline 2005 & -0.144 & & 1.171 & 0.152 & 3.774 & 0.017 & -0.733 & 0.610 & 0.366 & 0.610 & -0.155 & 0.441 & 0.268 & -0.033 \\
\hline 2006 & -0.140 & -1.147 & & 0.172 & & 0.023 & -0.751 & 0.712 & 0.383 & 0.570 & -0.277 & 0.185 & 0.288 & -0.081 \\
\hline 2007 & -0.127 & -0.908 & & 0.197 & & 0.008 & -0.680 & 0.619 & 0.293 & 0.586 & -0.499 & -0.034 & 0.281 & -0.143 \\
\hline 2008 & -0.068 & -0.560 & -0.100 & 0.207 & 2.300 & 0.009 & -0.588 & 0.496 & 0.003 & 0.697 & -0.428 & -0.356 & 0.255 & -0.157 \\
\hline 2009 & 0.000 & & 0.335 & 0.187 & 0.211 & 0.008 & -0.477 & 0.287 & -0.487 & & -0.226 & -0.906 & 0.175 & -0.085 \\
\hline 2010 & 0.045 & -0.165 & 0.515 & 0.173 & -1.149 & 0.015 & -0.452 & 0.153 & -0.829 & 0.151 & -0.079 & -0.603 & 0.141 & -0.013 \\
\hline 2011 & 0.105 & 0.487 & 1.696 & 0.130 & -2.332 & 0.031 & -0.445 & 0.030 & -0.836 & 0.035 & 0.166 & 0.137 & 0.097 & 0.083 \\
\hline 2012 & 0.153 & & 1.865 & 0.045 & & 0.049 & -0.458 & -0.191 & -0.701 & -0.142 & 0.588 & 0.833 & 0.065 & 0.176 \\
\hline 2013 & 0.172 & 1.594 & 2.385 & -0.068 & -4.004 & 0.083 & -0.393 & -0.294 & -0.635 & -0.066 & 0.904 & 1.676 & -0.054 & 0.217 \\
\hline
\end{tabular}

Source: Authors' calculation based on COMTRADE (2015) database 
Figure 2: Maturity index values of clutches and parts thereof for motor vehicles (870893) at national and global level, $1996-2013$

\begin{tabular}{|c|c|c|c|c|c|c|c|c|c|c|c|c|c|c|c|}
\hline Year & World & Algeria & Bahrain & China & Egypt & Germany & Iran & Jordan & Lebanon & Morocco & S. Arabia & S. Korea & Tunisia & Turkey & USA \\
\hline 1996 & -0.013 & 1.468 & & -0.022 & & 0.007 & & & & 0.368 & -0.113 & -0.084 & -0.074 & 0.280 & 0.005 \\
\hline 1997 & -0.014 & 1.524 & & 0.022 & & 0.021 & 1.892 & & -0.125 & 0.264 & & -0.066 & -0.031 & 0.321 & 0.052 \\
\hline 1998 & 0.000 & 1.386 & & 0.104 & & 0.016 & 2.006 & & 0.044 & 0.308 & 1.333 & -0.039 & -0.090 & 0.318 & 0.089 \\
\hline 1999 & 0.018 & 1.097 & & 0.143 & & 0.014 & 1.892 & 1.302 & 0.122 & 0.366 & 0.802 & -0.019 & -0.133 & 0.352 & 0.092 \\
\hline 2000 & 0.036 & 0.756 & -0.265 & 0.176 & & 0.014 & 1.448 & 1.114 & -0.050 & 0.384 & 0.261 & 0.005 & -0.006 & 0.427 & 0.104 \\
\hline 2001 & 0.044 & 0.242 & -0.187 & 0.178 & & 0.009 & 1.156 & & -0.123 & 0.341 & -0.509 & 0.017 & 0.034 & 0.358 & 0.092 \\
\hline 2002 & 0.042 & -0.117 & -0.166 & 0.192 & -0.432 & -0.006 & 0.937 & & -0.265 & 0.304 & 0.166 & 0.027 & 0.050 & 0.289 & 0.078 \\
\hline 2003 & 0.037 & -0.202 & -0.116 & 0.204 & -0.071 & -0.007 & 0.757 & 0.680 & -0.374 & 0.241 & -0.125 & 0.040 & 0.049 & 0.244 & 0.062 \\
\hline 2004 & 0.032 & -0.400 & -0.098 & 0.205 & & -0.001 & 0.615 & 0.638 & -0.458 & 0.178 & -0.303 & 0.052 & 0.046 & 0.207 & 0.043 \\
\hline 2005 & 0.023 & -0.599 & -0.198 & 0.169 & & 0.003 & 0.499 & 0.935 & -0.584 & 0.103 & -0.555 & 0.055 & -0.019 & 0.148 & 0.012 \\
\hline 2006 & 0.012 & -0.597 & -0.295 & 0.086 & & 0.016 & 0.341 & 0.990 & -0.713 & -0.014 & -0.452 & 0.056 & 0.028 & 0.131 & -0.010 \\
\hline 2007 & 0.000 & -0.457 & -0.231 & 0.039 & & 0.030 & & & -0.555 & -0.112 & -0.525 & 0.055 & 0.057 & 0.111 & -0.025 \\
\hline 2008 & -0.005 & -0.176 & -0.307 & 0.017 & 1.061 & 0.041 & & 0.944 & -0.405 & -0.107 & & 0.051 & 0.082 & 0.072 & -0.051 \\
\hline 2009 & 0.001 & 0.480 & -0.541 & 0.003 & 1.124 & 0.044 & & 0.858 & -0.080 & -0.053 & & 0.049 & 0.051 & -0.006 & -0.041 \\
\hline 2010 & 0.014 & 0.696 & -0.602 & -0.013 & 0.986 & 0.053 & -0.378 & 0.596 & 0.186 & -0.103 & 0.692 & 0.049 & 0.070 & -0.018 & -0.038 \\
\hline 2011 & 0.028 & & -0.735 & -0.027 & 0.908 & 0.043 & -0.849 & 0.263 & 0.529 & -0.110 & 0.724 & 0.044 & 0.121 & -0.048 & -0.035 \\
\hline 2012 & 0.047 & & -0.860 & -0.018 & 0.788 & 0.027 & & 0.145 & 0.809 & -0.133 & 0.842 & 0.037 & 0.282 & -0.070 & -0.023 \\
\hline 2013 & 0.066 & & -1.026 & 0.011 & 0.374 & 0.019 & & 0.167 & 0.961 & -0.212 & 0.540 & 0.064 & 0.776 & -0.074 & -0.011 \\
\hline
\end{tabular}

Source: Authors' calculation based on COMTRADE (2015) database 
Figure 3: Maturity index values of electric fans, motor $>125$ watts $(841459)$ at national and global level, $1996-2013$

\begin{tabular}{|c|c|c|c|c|c|c|c|c|c|c|c|c|c|c|c|}
\hline Year & World & Algeria & Bahrain & China & Egypt & Germany & Iran & Jordan & Lebanon & Morocco & S. Arabia & S. Korea & Tunisia & Turkey & USA \\
\hline 1996 & 0.026 & 0.672 & & -0.055 & 0.340 & 0.030 & & & & 1.027 & -0.690 & -0.094 & & -0.236 & -0.103 \\
\hline 1997 & 0.021 & 1.365 & & 0.056 & 0.556 & -0.002 & -0.594 & 1.343 & -0.047 & 0.940 & & -0.531 & & -0.219 & -0.074 \\
\hline 1998 & 0.014 & 2.102 & & 0.144 & 0.433 & -0.026 & -0.480 & 1.199 & -0.147 & 0.544 & -0.411 & -0.404 & & -0.196 & -0.060 \\
\hline 1999 & 0.007 & 1.180 & & 0.254 & 0.302 & -0.056 & -0.272 & 0.780 & -0.325 & 0.984 & -0.298 & -0.137 & & -0.136 & -0.043 \\
\hline 2000 & 0.002 & & 2.418 & 0.319 & 0.186 & -0.074 & 0.066 & 0.158 & -0.343 & 1.205 & -0.083 & 0.088 & & -0.009 & -0.011 \\
\hline 2001 & -0.006 & 0.434 & 1.488 & 0.243 & 0.260 & -0.063 & 0.207 & -0.105 & -0.297 & 1.157 & 0.089 & 0.238 & & 0.053 & 0.008 \\
\hline 2002 & -0.011 & 0.171 & 0.722 & 0.236 & 0.253 & -0.059 & 0.400 & -0.293 & -0.275 & 1.081 & 0.617 & 0.293 & & 0.113 & 0.019 \\
\hline 2003 & -0.015 & 0.072 & & 0.279 & 0.258 & -0.068 & 0.501 & -0.343 & -0.278 & 1.000 & 0.456 & 0.364 & 0.231 & 0.151 & 0.001 \\
\hline 2004 & -0.017 & -0.079 & -0.021 & 0.316 & 0.044 & -0.072 & 0.523 & -0.361 & -0.268 & 0.978 & 0.360 & 0.408 & & 0.149 & -0.013 \\
\hline 2005 & -0.017 & -0.245 & 0.082 & 0.291 & -0.145 & -0.059 & 0.445 & -0.271 & -0.246 & 0.968 & 0.193 & 0.457 & -0.137 & 0.097 & -0.022 \\
\hline 2006 & -0.014 & -0.299 & 0.235 & 0.295 & -0.216 & -0.052 & 0.292 & -0.194 & -0.261 & 1.064 & 0.012 & 0.352 & -0.111 & 0.097 & -0.025 \\
\hline 2007 & -0.008 & 0.074 & 0.410 & 0.364 & -0.277 & -0.048 & & -0.048 & -0.242 & 0.409 & -0.155 & 0.217 & -0.103 & 0.028 & -0.032 \\
\hline 2008 & -0.004 & 0.098 & 0.678 & 0.263 & -0.133 & -0.029 & & 0.118 & -0.146 & 0.012 & & 0.140 & 0.009 & -0.101 & -0.038 \\
\hline 2009 & 0.001 & 0.186 & 0.944 & 0.155 & -0.183 & -0.001 & & 0.338 & -0.036 & 0.080 & & 0.073 & 0.275 & -0.344 & 0.014 \\
\hline 2010 & 0.008 & 0.208 & 1.125 & 0.089 & -0.231 & 0.012 & -1.263 & 0.110 & 0.006 & 0.062 & -0.122 & 0.044 & 0.681 & -0.355 & 0.046 \\
\hline 2011 & 0.016 & 0.151 & 1.160 & -0.009 & -0.310 & 0.029 & -1.602 & -0.057 & 0.050 & 0.108 & -0.096 & -0.018 & & -0.307 & 0.092 \\
\hline 2012 & 0.026 & 0.301 & 1.351 & -0.130 & -0.450 & 0.030 & & -0.212 & 0.061 & 0.057 & -0.110 & -0.002 & & -0.177 & 0.123 \\
\hline 2013 & 0.035 & 0.321 & 1.764 & -0.246 & -0.677 & 0.035 & & -0.163 & 0.077 & 0.059 & 0.009 & 0.038 & & 0.012 & 0.130 \\
\hline
\end{tabular}

Source: Authors' calculation based on COMTRADE (2015) database 
Figure 4: Maturity index values of gaskets, washers and other seals of vulcanised rubber (401693) at national and global level, 1996-2013

\begin{tabular}{|c|c|c|c|c|c|c|c|c|c|c|c|c|c|c|c|}
\hline Year & World & Algeria & Bahrain & China & Egypt & Germany & Iran & Jordan & Lebanon & Morocco & Oman & S. Arabia & S. Korea & Tunisia & Turkey \\
\hline 1996 & 0.044 & 0.438 & & -0.053 & 0.590 & -0.040 & & & & 0.576 & & 0.469 & -0.032 & -0.235 & 0.118 \\
\hline 1997 & 0.039 & 0.600 & & -0.059 & 0.548 & -0.028 & 1.469 & 1.072 & 0.323 & 0.171 & & & 0.042 & -0.368 & 0.245 \\
\hline 1998 & 0.033 & 0.706 & & -0.026 & 0.132 & -0.017 & 1.347 & 0.810 & 0.542 & -0.066 & & 0.044 & 0.127 & -0.446 & 0.221 \\
\hline 1999 & 0.030 & 0.637 & & 0.010 & -0.306 & -0.006 & 1.095 & 0.238 & 0.640 & -0.010 & & -0.162 & 0.171 & -0.361 & 0.156 \\
\hline 2000 & 0.031 & 0.490 & -1.605 & 0.031 & -0.362 & 0.014 & 0.757 & -0.228 & 0.528 & 0.166 & & -0.275 & 0.192 & -0.157 & 0.138 \\
\hline 2001 & 0.026 & 0.304 & -1.118 & 0.032 & -0.150 & 0.020 & 0.435 & -0.599 & 0.459 & 0.296 & & -0.393 & 0.221 & -0.035 & 0.076 \\
\hline 2002 & 0.008 & 0.046 & -0.583 & 0.038 & 0.033 & 0.013 & 0.227 & -1.000 & 0.392 & 0.394 & 1.789 & -0.458 & 0.172 & 0.062 & 0.020 \\
\hline 2003 & -0.003 & -0.160 & -0.091 & 0.050 & 0.198 & 0.009 & 0.096 & -1.270 & 0.341 & 0.612 & 1.168 & -0.115 & 0.119 & 0.185 & 0.016 \\
\hline 2004 & -0.008 & -0.357 & 0.269 & 0.057 & 0.384 & 0.010 & 0.023 & -1.379 & 0.291 & 0.898 & 0.628 & 0.129 & 0.067 & 0.256 & 0.028 \\
\hline 2005 & -0.011 & -0.487 & 0.595 & 0.073 & 0.799 & 0.005 & 0.078 & -1.260 & -0.016 & 1.252 & -0.304 & 0.426 & -0.026 & 0.429 & -0.005 \\
\hline 2006 & -0.010 & -0.515 & 0.631 & 0.048 & 1.141 & 0.004 & 0.117 & -1.083 & -0.074 & 1.416 & -0.917 & 0.510 & -0.081 & 0.487 & 0.044 \\
\hline 2007 & -0.008 & -0.436 & 0.519 & 0.013 & 1.421 & 0.007 & & -0.886 & -0.128 & 1.330 & -1.152 & 0.693 & -0.119 & 0.454 & 0.068 \\
\hline 2008 & -0.008 & -0.291 & 0.359 & -0.009 & 1.486 & 0.014 & & -0.685 & -0.216 & 1.118 & -1.120 & & -0.129 & 0.362 & 0.064 \\
\hline 2009 & -0.001 & -0.067 & -0.072 & -0.003 & 1.215 & 0.018 & & -0.403 & -0.305 & 0.843 & -0.528 & & -0.100 & 0.135 & 0.033 \\
\hline 2010 & 0.018 & 0.316 & -0.467 & 0.005 & 0.957 & 0.033 & 0.007 & 0.239 & -0.439 & 0.634 & -0.759 & 1.027 & -0.045 & -0.020 & 0.025 \\
\hline 2011 & 0.030 & 0.592 & -0.776 & 0.011 & 0.797 & 0.040 & 0.049 & 0.571 & -0.586 & 0.112 & -0.383 & 0.924 & 0.039 & -0.201 & -0.016 \\
\hline 2012 & 0.039 & 0.923 & -0.908 & 0.030 & 0.838 & 0.040 & & 0.776 & -0.608 & -0.659 & 0.048 & 0.704 & 0.201 & -0.361 & -0.073 \\
\hline 2013 & 0.048 & 0.857 & -0.730 & 0.048 & 0.496 & 0.051 & & 0.950 & -0.602 & -1.219 & 1.003 & 0.040 & 0.429 & -0.676 & -0.116 \\
\hline
\end{tabular}

Source: Authors' calculation based on COMTRADE (2015) database 
Figure 5: Maturity index values of parts of power transmission etc equipment (848390) at national and global level, $1996-2013$

\begin{tabular}{|c|c|c|c|c|c|c|c|c|c|c|c|c|c|c|c|}
\hline Year & World & Algeria & Bahrain & China & Egypt & Germany & Iran & Jordan & Lebanon & Morocco & S. Arabia & S. Korea & Tunisia & Turkey & USA \\
\hline 1996 & -0.035 & & & 0.238 & & -0.119 & & & & -0.715 & -0.437 & 0.479 & -1.053 & 0.098 & -0.099 \\
\hline 1997 & -0.043 & & & 0.252 & & -0.096 & 0.018 & -0.256 & -0.117 & -0.534 & & 0.433 & -0.078 & 0.295 & -0.095 \\
\hline 1998 & -0.035 & 1.828 & & 0.215 & -2.196 & -0.071 & -0.130 & & 0.544 & -0.901 & 0.370 & 0.377 & 0.638 & 0.328 & -0.075 \\
\hline 1999 & -0.019 & & & 0.171 & -0.243 & -0.043 & 0.031 & -0.501 & 1.130 & -0.240 & 0.379 & 0.302 & 1.006 & 0.405 & -0.048 \\
\hline 2000 & 0.000 & 0.450 & 1.286 & 0.149 & 0.879 & -0.011 & 0.614 & -1.009 & 1.947 & 0.242 & 0.403 & 0.208 & 1.375 & 0.430 & 0.000 \\
\hline 2001 & 0.008 & -0.075 & 1.274 & 0.111 & & 0.000 & 0.515 & -1.171 & 2.585 & 0.565 & 0.479 & 0.124 & 1.504 & 0.362 & 0.028 \\
\hline 2002 & 0.010 & -0.495 & 0.974 & 0.065 & 2.370 & 0.009 & 0.064 & -1.236 & 2.785 & 0.986 & 0.092 & 0.091 & 1.596 & 0.278 & 0.049 \\
\hline 2003 & 0.012 & -0.539 & & 0.041 & & 0.019 & -0.418 & -1.093 & 2.846 & 1.437 & 0.008 & 0.117 & 1.649 & 0.230 & 0.049 \\
\hline 2004 & 0.013 & -0.632 & 0.883 & 0.038 & 3.793 & 0.027 & -0.770 & -0.837 & 2.907 & 1.711 & 0.077 & 0.142 & 1.701 & 0.203 & 0.048 \\
\hline 2005 & 0.015 & -0.633 & & 0.022 & 3.250 & 0.025 & -0.832 & -0.527 & 2.252 & 1.949 & 0.095 & 0.187 & 1.228 & 0.101 & 0.048 \\
\hline 2006 & 0.012 & -0.699 & & 0.066 & 2.235 & 0.021 & -1.178 & -0.324 & 1.946 & 2.318 & -0.141 & 0.229 & 0.959 & 0.099 & 0.040 \\
\hline 2007 & 0.006 & & & 0.109 & 1.848 & 0.021 & & -0.001 & 1.706 & 1.612 & 0.044 & 0.285 & 0.727 & 0.101 & 0.023 \\
\hline 2008 & 0.003 & -0.614 & 0.055 & 0.150 & 1.014 & 0.022 & & 0.307 & 1.416 & 0.829 & & 0.326 & 0.406 & 0.033 & -0.004 \\
\hline 2009 & 0.001 & -0.351 & 0.367 & 0.136 & 0.520 & 0.010 & & 0.586 & 1.010 & 0.146 & & 0.318 & -0.145 & -0.015 & 0.024 \\
\hline 2010 & 0.009 & & 0.584 & 0.156 & 0.079 & 0.005 & -1.882 & 0.596 & 0.794 & -0.202 & 1.467 & 0.305 & -0.474 & 0.018 & 0.031 \\
\hline 2011 & 0.018 & -1.060 & 0.314 & 0.164 & -0.532 & -0.002 & -3.345 & 0.469 & 0.407 & -0.515 & 1.701 & 0.247 & -0.746 & 0.020 & 0.049 \\
\hline 2012 & 0.028 & -1.030 & 0.284 & 0.139 & -0.695 & -0.008 & & 0.205 & -0.319 & -0.527 & 1.802 & 0.182 & -0.993 & 0.008 & 0.055 \\
\hline 2013 & 0.030 & -0.365 & 0.863 & 0.088 & -0.686 & -0.006 & & 0.277 & -0.623 & -0.448 & 1.080 & 0.117 & -0.693 & -0.014 & 0.045 \\
\hline
\end{tabular}

Source: Authors' calculation based on COMTRADE (2015) database 
Figure 6: Maturity index values of ignition/other wiring sets for vehicles/aircraft/ship (854430) at national and global level, 1996-2013

\begin{tabular}{|c|c|c|c|c|c|c|c|c|c|c|c|c|c|c|c|}
\hline Year & World & Bahrain & China & Egypt & Germany & Iran & Jordan & Lebanon & Morocco & Oman & S. Arabia & S. Korea & Tunisia & Turkey & USA \\
\hline 1996 & 0.059 & & 0.102 & & 0.014 & & & & -2.047 & & 3.462 & 0.400 & 1.778 & 0.969 & -0.099 \\
\hline 1997 & 0.040 & & 0.109 & & 0.038 & 0.153 & & 0.445 & -1.795 & & & 0.436 & 0.887 & 0.407 & -0.114 \\
\hline 1998 & 0.030 & & 0.112 & & 0.042 & -0.120 & & -0.789 & & & & 0.552 & 0.056 & -0.059 & -0.105 \\
\hline 1999 & 0.024 & & 0.095 & & 0.063 & 0.039 & -2.875 & -1.466 & 0.212 & 1.382 & 0.885 & 0.646 & -0.210 & -0.209 & -0.077 \\
\hline 2000 & 0.026 & & 0.076 & -1.257 & 0.095 & 0.635 & & & -0.080 & & 0.315 & 0.697 & -0.321 & -0.214 & -0.030 \\
\hline 2001 & 0.021 & & 0.050 & -1.320 & 0.093 & 0.856 & & -2.244 & -0.384 & & -0.745 & 0.743 & -0.381 & -0.312 & -0.007 \\
\hline 2002 & 0.001 & & 0.040 & -1.222 & 0.062 & 0.610 & & -2.324 & -0.826 & -0.658 & -1.104 & 0.672 & -0.341 & -0.130 & -0.016 \\
\hline 2003 & -0.012 & 2.949 & 0.053 & -1.060 & 0.017 & 0.362 & & -2.437 & -1.220 & -0.526 & -3.750 & 0.575 & -0.359 & 0.083 & -0.037 \\
\hline 2004 & -0.017 & 2.730 & 0.059 & & -0.013 & 0.119 & 0.261 & -2.623 & -1.479 & -0.362 & -2.450 & 0.486 & -0.432 & 0.214 & -0.053 \\
\hline 2005 & -0.018 & 2.204 & 0.050 & & -0.054 & -0.133 & 0.530 & & -1.979 & 0.276 & -1.151 & 0.340 & -0.110 & 0.555 & -0.068 \\
\hline 2006 & -0.012 & & 0.055 & & -0.068 & 0.166 & 0.627 & -0.986 & -2.958 & 0.525 & 1.393 & 0.195 & 0.015 & 0.735 & -0.078 \\
\hline 2007 & -0.008 & & 0.008 & 1.221 & -0.083 & & & -0.817 & -3.200 & & 3.900 & 0.062 & 0.039 & 0.594 & -0.100 \\
\hline 2008 & -0.011 & 0.761 & -0.037 & & -0.073 & & 1.199 & -0.568 & -1.583 & 1.170 & & -0.018 & 0.093 & 0.381 & -0.124 \\
\hline 2009 & -0.004 & & -0.048 & 0.684 & -0.070 & & -0.329 & -0.140 & 0.913 & 0.621 & & -0.054 & 0.163 & 0.162 & -0.079 \\
\hline 2010 & 0.026 & -0.901 & -0.059 & 0.048 & -0.015 & 5.260 & -0.569 & -0.046 & 1.061 & & & -0.067 & 0.081 & -0.072 & -0.034 \\
\hline 2011 & 0.051 & -0.901 & -0.083 & -0.310 & 0.047 & 8.342 & -0.438 & 0.227 & 1.080 & -1.494 & & -0.061 & 0.065 & -0.301 & 0.015 \\
\hline 2012 & 0.074 & -0.562 & -0.098 & -0.604 & 0.091 & & -0.685 & 0.499 & 0.915 & -1.316 & & -0.044 & 0.075 & -0.427 & 0.058 \\
\hline 2013 & 0.099 & -0.148 & -0.109 & 0.001 & 0.148 & & -0.946 & 0.004 & 0.854 & -1.216 & & 0.017 & 0.014 & -0.511 & 0.089 \\
\hline
\end{tabular}

Source: Authors' calculation based on COMTRADE (2015) database 
Figure 7: Maturity index values of ignition/other wiring sets for vehicles/aircraft/ship (854430) at national and global level, 1996-2013

\begin{tabular}{|c|c|c|c|c|c|c|c|c|c|c|c|c|c|c|c|}
\hline Year & World & Bahrain & China & Egypt & Germany & Iran & Jordan & Lebanon & Morocco & Oman & S. Arabia & S. Korea & Tunisia & Turkey & USA \\
\hline 1996 & -0.010 & & -0.059 & 0.959 & 0.093 & & & & -0.138 & & 0.244 & 0.084 & 0.297 & 0.231 & -0.026 \\
\hline 1997 & -0.009 & & -0.105 & 1.080 & 0.094 & 0.224 & 0.906 & 0.780 & 0.057 & & & 0.140 & 0.424 & 0.118 & -0.017 \\
\hline 1998 & 0.000 & & -0.169 & 0.451 & 0.075 & 0.160 & 0.608 & 0.178 & -0.032 & & 0.109 & 0.172 & 0.368 & 0.032 & -0.006 \\
\hline 1999 & 0.010 & & -0.222 & -0.127 & 0.059 & 0.023 & 0.313 & -0.165 & -0.175 & & 0.032 & 0.171 & 0.335 & 0.007 & 0.005 \\
\hline 2000 & 0.021 & & -0.210 & -0.403 & 0.053 & -0.086 & -0.079 & -0.199 & -0.213 & 0.038 & -0.083 & 0.141 & 0.306 & 0.007 & 0.012 \\
\hline 2001 & 0.027 & & -0.072 & -0.589 & 0.037 & -0.150 & -0.239 & -0.220 & -0.170 & & -0.035 & 0.128 & 0.291 & 0.066 & 0.015 \\
\hline 2002 & 0.027 & -0.717 & -0.036 & -0.690 & 0.015 & 0.010 & -0.323 & -0.169 & -0.076 & 0.934 & -0.141 & 0.100 & 0.288 & 0.109 & 0.010 \\
\hline 2003 & 0.025 & 0.184 & -0.092 & -0.757 & -0.006 & 0.209 & -0.362 & -0.123 & -0.099 & 1.223 & -0.331 & 0.077 & 0.313 & 0.126 & 0.006 \\
\hline 2004 & 0.023 & 0.878 & -0.136 & -0.769 & -0.018 & 0.437 & -0.383 & -0.077 & -0.154 & 1.260 & -0.426 & 0.060 & 0.340 & 0.121 & 0.005 \\
\hline 2005 & 0.018 & & -0.136 & -0.786 & -0.028 & 0.597 & -0.248 & 0.268 & -0.248 & 1.224 & -0.542 & 0.031 & 0.334 & 0.160 & 0.007 \\
\hline 2006 & 0.011 & 1.113 & -0.243 & -0.567 & -0.021 & 0.455 & -0.121 & 0.345 & -0.203 & 1.006 & -0.644 & 0.012 & 0.356 & 0.152 & 0.008 \\
\hline 2007 & 0.004 & 1.025 & -0.170 & -0.544 & -0.023 & & 0.063 & 0.321 & -0.169 & 0.761 & -0.739 & 0.009 & 0.337 & 0.128 & 0.010 \\
\hline 2008 & -0.002 & 0.609 & -0.036 & -0.717 & -0.021 & & 0.294 & 0.261 & -0.246 & 0.254 & -0.743 & 0.015 & 0.304 & 0.115 & 0.020 \\
\hline 2009 & -0.001 & 0.785 & 0.082 & -0.944 & -0.015 & & 0.650 & 0.120 & -0.603 & -0.621 & -0.477 & 0.016 & 0.244 & 0.113 & 0.041 \\
\hline 2010 & 0.003 & 0.693 & 0.150 & -1.159 & 0.010 & -2.200 & 0.795 & 0.032 & -0.728 & -0.997 & -0.234 & 0.016 & 0.223 & 0.085 & 0.061 \\
\hline 2011 & 0.008 & 0.723 & 0.264 & -1.050 & 0.033 & -4.081 & 0.930 & -0.064 & -0.651 & -1.259 & 0.010 & 0.012 & 0.176 & 0.070 & 0.074 \\
\hline 2012 & 0.014 & 0.734 & 0.375 & -1.032 & 0.046 & & 1.102 & -0.155 & -0.529 & -1.479 & 0.238 & 0.002 & 0.104 & 0.080 & 0.079 \\
\hline 2013 & 0.026 & 0.436 & 0.411 & -0.931 & 0.060 & & 1.214 & -0.239 & -0.138 & -1.311 & 0.443 & -0.014 & 0.046 & 0.079 & 0.077 \\
\hline
\end{tabular}

Source: Authors' calculation based on COMTRADE (2015) database 
Figure 8: Maturity index values of articles of vulcanised rubber nes (401699) at national and global level, 1996-2013

\begin{tabular}{|c|c|c|c|c|c|c|c|c|c|c|c|c|c|c|c|}
\hline Year & World & Algeria & Bahrain & China & Egypt & Germany & Iran & Jordan & Lebanon & Morocco & Oman & S. Arabia & S. Korea & Tunisia & Turkey \\
\hline 1996 & 0.023 & 0.528 & & 0.065 & 0.838 & 0.025 & & & & -0.228 & & 0.594 & 0.042 & -0.393 & 0.209 \\
\hline 1997 & 0.014 & 0.935 & & 0.001 & 0.668 & -0.008 & 1.968 & 0.666 & -0.113 & -0.253 & & & 0.079 & -0.125 & 0.230 \\
\hline 1998 & 0.011 & 0.600 & & -0.021 & 0.056 & -0.027 & 1.415 & 0.637 & -0.071 & -0.067 & & 0.122 & 0.099 & 0.110 & 0.138 \\
\hline 1999 & 0.011 & 0.520 & & -0.042 & -0.184 & -0.033 & 1.134 & 0.386 & -0.023 & -0.006 & & -0.042 & 0.098 & 0.447 & 0.102 \\
\hline 2000 & 0.014 & 0.459 & 1.800 & -0.043 & -0.348 & -0.019 & 0.900 & 0.004 & 0.033 & 0.141 & & -0.130 & 0.100 & 0.675 & 0.093 \\
\hline 2001 & 0.013 & 0.371 & & -0.026 & -0.499 & -0.014 & 0.728 & -0.209 & 0.087 & 0.285 & & -0.151 & 0.093 & 0.782 & 0.107 \\
\hline 2002 & 0.001 & 0.269 & 1.078 & -0.007 & -0.571 & -0.028 & 0.555 & -0.317 & 0.143 & 0.401 & -0.154 & -0.662 & 0.049 & 0.840 & 0.085 \\
\hline 2003 & -0.008 & 0.280 & & 0.002 & -0.621 & -0.037 & 0.425 & -0.310 & 0.166 & 0.414 & -0.034 & -0.337 & 0.022 & 0.819 & 0.063 \\
\hline 2004 & -0.013 & 0.108 & 0.442 & 0.006 & -0.639 & -0.039 & 0.277 & -0.365 & 0.185 & 0.401 & 0.096 & -0.217 & 0.008 & 0.755 & 0.048 \\
\hline 2005 & -0.016 & -0.081 & 0.096 & 0.038 & -0.544 & -0.025 & 0.190 & -0.345 & 0.166 & 0.404 & 0.228 & -0.130 & -0.013 & 0.553 & 0.041 \\
\hline 2006 & -0.016 & -0.148 & -0.229 & 0.011 & -0.330 & -0.015 & 0.256 & -0.267 & 0.168 & 0.369 & 0.216 & -0.058 & -0.009 & 0.424 & 0.073 \\
\hline 2007 & -0.014 & -0.299 & -0.301 & -0.002 & -0.393 & -0.002 & & -0.190 & 0.158 & 0.468 & 0.071 & 0.067 & -0.004 & 0.282 & 0.075 \\
\hline 2008 & -0.010 & -0.369 & -0.095 & 0.003 & -0.539 & 0.012 & & -0.149 & 0.118 & 0.595 & -0.245 & & -0.006 & 0.094 & 0.071 \\
\hline 2009 & -0.003 & -0.196 & 0.216 & 0.028 & -0.564 & 0.024 & & -0.129 & 0.067 & 0.411 & -0.877 & & 0.009 & -0.182 & 0.070 \\
\hline 2010 & 0.017 & -0.307 & 0.382 & 0.044 & -0.506 & 0.047 & 1.372 & -0.168 & 0.038 & 0.492 & -1.142 & -0.684 & 0.051 & -0.238 & 0.069 \\
\hline 2011 & 0.033 & -0.361 & 0.691 & 0.073 & -0.152 & 0.059 & 2.316 & -0.180 & 0.038 & 0.615 & -0.890 & -0.770 & 0.085 & -0.245 & 0.057 \\
\hline 2012 & 0.048 & 0.053 & 1.125 & 0.125 & -0.502 & 0.059 & & 0.036 & 0.033 & 0.666 & -0.496 & -0.916 & 0.116 & -0.227 & 0.031 \\
\hline 2013 & 0.064 & 0.321 & 1.604 & 0.237 & -0.457 & 0.060 & & 0.079 & -0.005 & 0.546 & -0.213 & -1.004 & 0.135 & -0.120 & 0.000 \\
\hline
\end{tabular}

Source: Authors' calculation based on COMTRADE (2015) database 


\section{Figure 9: Maturity index values of parts of taps, cocks, valves or similar appliances (848190) at national and global level, 1996-2013}

\begin{tabular}{|c|c|c|c|c|c|c|c|c|c|c|c|c|c|c|}
\hline Year & World & Algeria & Bahrain & China & Egypt & Germany & Iran & Jordan & Lebanon & Morocco & Oman & S. Arabia & S. Korea & Tunisia \\
\hline 1996 & 0.024 & 0.222 & & 0.371 & & -0.029 & & & & -0.227 & 1.077 & -0.339 & 0.132 & -0.759 \\
\hline 1997 & 0.020 & -0.205 & & 0.416 & -0.483 & -0.030 & 1.044 & 0.055 & -0.617 & 0.341 & 1.033 & & 0.161 & -0.647 \\
\hline 1998 & 0.025 & -0.617 & & 0.425 & -0.101 & -0.033 & 0.924 & & 0.146 & 0.360 & & -0.027 & 0.187 & -0.429 \\
\hline 1999 & 0.037 & -0.492 & & 0.409 & 0.131 & -0.028 & 0.894 & -0.027 & 0.855 & 0.819 & & -0.002 & 0.198 & -0.271 \\
\hline 2000 & 0.049 & -0.302 & & 0.367 & 0.421 & -0.014 & 0.478 & -0.005 & 1.139 & 0.976 & & -0.047 & 0.197 & 0.108 \\
\hline 2001 & 0.048 & & & 0.274 & 0.418 & -0.001 & 0.261 & 0.040 & 1.231 & 0.832 & -0.298 & -0.017 & 0.179 & 0.383 \\
\hline 2002 & 0.046 & 0.085 & & 0.184 & 0.447 & 0.010 & 0.153 & 0.032 & 1.203 & 0.736 & 0.506 & -0.177 & 0.154 & 0.631 \\
\hline 2003 & 0.046 & 0.226 & 1.637 & 0.124 & 0.460 & 0.022 & 0.077 & 0.114 & 1.154 & 0.706 & 1.074 & -0.144 & 0.122 & 0.718 \\
\hline 2004 & 0.044 & 0.270 & & 0.083 & 0.424 & 0.034 & -0.003 & 0.190 & 1.134 & 0.658 & 1.703 & -0.016 & 0.091 & 0.673 \\
\hline 2005 & 0.038 & 0.365 & 1.566 & -0.003 & 0.372 & 0.048 & -0.120 & 0.304 & 0.673 & 0.486 & & 0.007 & 0.040 & 0.575 \\
\hline 2006 & 0.033 & 0.400 & & 0.028 & 0.291 & 0.056 & -0.048 & 0.383 & 0.558 & 0.371 & 2.943 & 0.006 & 0.005 & 0.463 \\
\hline 2007 & 0.022 & 0.248 & & 0.008 & 0.314 & 0.054 & & 0.389 & 0.443 & -0.162 & 3.085 & -0.034 & -0.030 & 0.346 \\
\hline 2008 & 0.009 & 0.103 & 1.274 & -0.038 & 0.398 & 0.049 & & 0.418 & 0.274 & -0.217 & 3.105 & & -0.061 & 0.267 \\
\hline 2009 & -0.002 & 0.073 & 1.194 & -0.079 & 0.408 & 0.041 & & 0.427 & 0.058 & 0.463 & 2.713 & & -0.086 & 0.002 \\
\hline 2010 & 0.000 & 0.023 & 0.827 & -0.072 & 0.222 & 0.027 & 0.851 & 0.338 & -0.005 & 0.342 & 1.726 & 0.045 & -0.090 & -0.041 \\
\hline 2011 & 0.003 & -0.039 & 0.891 & -0.072 & 0.197 & 0.007 & 1.702 & 0.089 & -0.084 & 0.314 & 1.439 & 0.061 & -0.083 & 0.051 \\
\hline 2012 & 0.014 & 0.028 & 1.740 & -0.086 & 0.178 & -0.020 & & -0.160 & -0.340 & 0.392 & 1.067 & 0.002 & -0.066 & 0.267 \\
\hline 2013 & 0.035 & 0.033 & 3.010 & -0.105 & -0.099 & -0.043 & & -0.566 & -0.580 & 0.396 & 1.022 & 0.030 & -0.033 & 0.451 \\
\hline
\end{tabular}

Source: Authors' calculation based on COMTRADE (2015) database 
Figure 10: Maturity level of parts of vacuum pumps, compressors, fans, blowers, hoods (841490) at national and global level, 1996-2013

\begin{tabular}{|c|c|c|c|c|c|c|c|c|c|c|c|c|c|c|}
\hline Year & World & Algeria & Bahrain & China & Egypt & Germany & Iran & Jordan & Lebanon & Morocco & Oman & S. Arabia & S. Korea & Tunisia \\
\hline 1996 & -0.020 & 1.875 & & 0.041 & 0.717 & -0.168 & & & & 2.002 & 0.302 & 0.703 & 0.610 & -0.375 \\
\hline 1997 & -0.010 & 0.727 & & 0.120 & 0.755 & -0.123 & 0.201 & 0.543 & 2.119 & 0.453 & 0.433 & & 0.572 & 0.025 \\
\hline 1998 & 0.002 & -0.223 & & 0.166 & 0.236 & -0.069 & 0.203 & & 0.971 & -0.130 & 0.738 & 0.337 & 0.446 & 0.123 \\
\hline 1999 & 0.017 & -0.818 & & 0.189 & & -0.027 & -0.007 & 0.237 & 0.095 & -0.416 & 0.584 & 0.396 & 0.327 & -0.021 \\
\hline 2000 & 0.032 & -0.912 & -0.129 & 0.214 & 0.347 & 0.011 & -0.287 & -0.113 & -0.587 & -0.510 & 0.402 & 0.483 & 0.253 & -0.345 \\
\hline 2001 & 0.032 & -0.889 & -0.182 & 0.172 & 0.316 & 0.047 & -0.270 & -0.310 & -1.120 & -0.484 & 0.274 & 0.515 & 0.168 & -0.618 \\
\hline 2002 & 0.034 & -0.563 & -0.237 & 0.145 & 0.314 & 0.083 & -0.168 & -0.485 & -1.162 & -0.455 & 0.055 & 0.240 & 0.092 & -0.749 \\
\hline 2003 & 0.034 & -0.279 & -0.113 & 0.138 & 0.384 & 0.094 & -0.064 & -0.550 & -1.208 & -0.319 & -0.244 & 0.396 & 0.045 & -0.819 \\
\hline 2004 & 0.035 & -0.128 & -0.013 & 0.140 & 0.535 & 0.093 & -0.112 & -0.581 & -1.202 & -0.225 & -0.604 & 0.372 & 0.020 & -0.860 \\
\hline 2005 & 0.028 & 0.199 & 0.244 & 0.096 & 0.613 & 0.072 & 0.056 & -0.578 & -0.308 & 0.042 & -0.763 & 0.438 & 0.025 & -0.897 \\
\hline 2006 & 0.021 & 0.418 & 0.334 & 0.098 & 0.812 & 0.044 & 0.230 & -0.490 & -0.082 & 0.193 & -0.846 & 0.555 & 0.069 & -0.914 \\
\hline 2007 & 0.012 & 0.439 & 0.479 & 0.100 & 0.679 & 0.032 & & -0.392 & -0.068 & 0.227 & -0.856 & 0.699 & 0.111 & -0.716 \\
\hline 2008 & 0.002 & 0.139 & 0.426 & 0.091 & 0.511 & 0.034 & & -0.247 & -0.020 & -0.003 & -0.856 & & 0.117 & -0.439 \\
\hline 2009 & -0.001 & -0.103 & 0.165 & 0.066 & 0.467 & 0.021 & & -0.085 & -0.003 & -0.426 & -0.660 & & 0.107 & 0.158 \\
\hline 2010 & 0.001 & -0.390 & 0.085 & 0.075 & 0.218 & -0.005 & 0.970 & 0.086 & -0.155 & -0.457 & -0.194 & 0.135 & 0.135 & 0.146 \\
\hline 2011 & 0.002 & -0.459 & -0.141 & 0.073 & -0.170 & -0.008 & 2.146 & 0.222 & -0.225 & -0.655 & -0.042 & 0.092 & 0.152 & 0.074 \\
\hline 2012 & 0.005 & -0.418 & -0.223 & 0.056 & -0.519 & -0.004 & & 0.420 & -0.259 & -0.673 & 0.210 & 0.129 & 0.132 & -0.051 \\
\hline 2013 & 0.014 & & -0.102 & 0.020 & -0.471 & -0.010 & & 0.623 & -0.086 & -0.475 & 0.050 & 0.261 & 0.116 & -0.495 \\
\hline
\end{tabular}

Source: Authors' calculation based on COMTRADE (2015) database 
Figure 11: Maturity level of mens, boys shirts, of cotton (620520) at national and global level, 1996-2013

\begin{tabular}{|c|c|c|c|c|c|c|c|c|c|c|c|c|c|c|}
\hline Year & World & Bahrain & China & Egypt & Germany & Iran & Jordan & Lebanon & Morocco & Oman & S. Arabia & S. Korea & Tunisia & Turkey \\
\hline 1996 & -0.060 & & -0.013 & 0.075 & -0.035 & & & & -0.036 & & -0.030 & -0.171 & -0.067 & -0.263 \\
\hline 1997 & -0.063 & & -0.040 & 0.055 & -0.072 & 0.122 & 1.497 & -0.588 & -0.008 & & & -0.186 & -0.066 & -0.162 \\
\hline 1998 & -0.062 & & -0.054 & -0.058 & -0.073 & -0.112 & & -0.246 & -0.048 & & 1.126 & -0.147 & -0.077 & -0.133 \\
\hline 1999 & -0.052 & & -0.068 & -0.130 & -0.054 & -0.958 & 2.418 & -0.044 & -0.024 & & 1.189 & -0.085 & -0.099 & -0.111 \\
\hline 2000 & -0.041 & -1.606 & -0.087 & -0.126 & -0.017 & -1.569 & 1.793 & 0.190 & -0.008 & -0.311 & 1.053 & -0.063 & -0.098 & -0.069 \\
\hline 2001 & -0.032 & -1.902 & -0.099 & -0.111 & 0.003 & -1.417 & & 0.374 & -0.005 & -0.360 & 0.775 & -0.017 & -0.094 & -0.015 \\
\hline 2002 & -0.022 & -1.925 & -0.065 & -0.085 & 0.030 & -1.077 & 0.879 & 0.431 & -0.012 & -0.226 & 1.464 & -0.113 & -0.083 & -0.030 \\
\hline 2003 & -0.021 & -1.623 & -0.033 & -0.041 & 0.049 & -0.582 & 0.243 & 0.440 & -0.039 & 0.072 & 1.642 & -0.227 & -0.088 & -0.063 \\
\hline 2004 & -0.018 & -1.229 & -0.005 & 0.013 & 0.060 & -0.111 & -0.145 & 0.429 & -0.064 & 0.014 & 2.629 & -0.318 & -0.097 & -0.086 \\
\hline 2005 & -0.013 & 0.063 & 0.032 & 0.187 & 0.084 & 0.827 & -0.793 & 0.119 & -0.074 & 0.325 & 3.483 & -0.430 & -0.105 & -0.128 \\
\hline 2006 & -0.011 & 0.682 & 0.041 & 0.328 & 0.080 & 1.504 & -1.089 & 0.014 & -0.062 & & 3.772 & -0.515 & -0.116 & -0.129 \\
\hline 2007 & -0.011 & 0.799 & 0.030 & 0.462 & 0.074 & & -1.202 & -0.058 & -0.104 & & 4.010 & -0.585 & -0.127 & -0.106 \\
\hline 2008 & -0.009 & 0.808 & 0.023 & 0.592 & 0.066 & & -0.999 & -0.085 & -0.089 & 1.173 & & -0.568 & -0.133 & -0.083 \\
\hline 2009 & -0.001 & 0.406 & 0.028 & 0.694 & 0.047 & & -0.512 & -0.133 & -0.096 & 1.295 & & -0.483 & -0.148 & -0.060 \\
\hline 2010 & 0.004 & 0.174 & 0.012 & 0.641 & 0.020 & 3.593 & -0.179 & -0.073 & -0.090 & 1.145 & & -0.378 & -0.181 & -0.027 \\
\hline 2011 & 0.016 & -0.174 & -0.008 & 0.479 & 0.001 & & 0.171 & 0.028 & -0.059 & 1.216 & 4.835 & -0.226 & -0.186 & 0.005 \\
\hline 2012 & 0.018 & -0.258 & -0.033 & 0.213 & -0.018 & & 0.263 & 0.071 & -0.041 & 0.633 & 4.841 & -0.023 & -0.199 & 0.020 \\
\hline 2013 & 0.021 & 0.413 & -0.044 & 0.006 & -0.038 & & 0.879 & 0.159 & -0.032 & 0.109 & & 0.183 & -0.224 & 0.016 \\
\hline
\end{tabular}

Source: Authors' calculation based on COMTRADE (2015) database 
Figure 12: Maturity level of moulds, injection \& compression, for rubber or plastic (848071) at national and global level, $1996-2013$

\begin{tabular}{|c|c|c|c|c|c|c|c|c|c|c|c|c|c|c|}
\hline Year & World & Algeria & China & Egypt & Germany & Iran & Jordan & Lebanon & Morocco & S. Arabia & S. Korea & Tunisia & Turkey & USA \\
\hline 1996 & -0.012 & & 0.274 & -0.370 & -0.007 & & & & 0.491 & 1.969 & 0.037 & 1.418 & 0.208 & 0.070 \\
\hline 1997 & -0.007 & 1.527 & 0.261 & -0.020 & -0.022 & -0.344 & 0.247 & 0.029 & 0.317 & & 0.042 & 0.648 & 0.212 & 0.038 \\
\hline 1998 & -0.004 & 0.022 & 0.265 & & -0.042 & -0.078 & -0.187 & -0.689 & 0.687 & & 0.060 & 0.210 & 0.071 & 0.007 \\
\hline 1999 & 0.002 & -0.461 & 0.238 & 0.205 & -0.047 & 0.160 & -0.322 & -0.889 & 0.692 & 0.554 & 0.064 & -0.085 & -0.002 & 0.000 \\
\hline 2000 & 0.002 & -0.828 & 0.233 & & -0.034 & 0.426 & -0.498 & -0.811 & 0.696 & 0.171 & 0.047 & -0.252 & -0.067 & -0.009 \\
\hline 2001 & 0.011 & -0.744 & 0.223 & & -0.009 & 0.759 & -0.562 & -0.740 & 0.695 & 0.078 & 0.024 & -0.338 & -0.052 & -0.030 \\
\hline 2002 & 0.012 & & 0.238 & -0.021 & -0.003 & 0.569 & -0.559 & -0.486 & 0.615 & -0.647 & -0.027 & -0.331 & -0.004 & -0.018 \\
\hline 2003 & -0.003 & -4.725 & 0.244 & & -0.023 & 0.313 & -0.489 & -0.315 & 0.718 & -1.223 & -0.076 & -0.247 & -0.004 & -0.022 \\
\hline 2004 & -0.018 & & 0.235 & & -0.044 & 0.156 & -0.455 & -0.279 & 0.713 & -0.876 & -0.109 & -0.156 & -0.017 & -0.043 \\
\hline 2005 & -0.033 & & 0.221 & -0.335 & -0.054 & -0.088 & -0.337 & 0.285 & 0.601 & -0.462 & -0.146 & 0.146 & -0.020 & -0.054 \\
\hline 2006 & -0.039 & -1.873 & 0.188 & & -0.057 & -0.191 & -0.268 & 0.353 & 0.299 & -0.310 & -0.157 & 0.294 & 0.033 & -0.062 \\
\hline 2007 & -0.043 & -0.176 & 0.151 & -0.363 & -0.069 & & -0.225 & 0.240 & 0.332 & -0.013 & -0.154 & 0.308 & -0.016 & -0.072 \\
\hline 2008 & -0.027 & & 0.136 & -0.419 & -0.055 & & -0.120 & 0.076 & 0.280 & & -0.120 & 0.261 & -0.006 & -0.058 \\
\hline 2009 & -0.007 & 5.899 & 0.113 & & -0.041 & & 0.032 & -0.141 & 0.217 & 0.050 & -0.071 & 0.185 & 0.035 & 0.001 \\
\hline 2010 & 0.011 & & 0.085 & -0.281 & -0.022 & 0.975 & 0.098 & -0.350 & -0.010 & & -0.001 & 0.087 & 0.064 & -0.008 \\
\hline 2011 & 0.047 & & 0.070 & -0.152 & 0.019 & 1.118 & 0.161 & -0.446 & -0.370 & & 0.086 & -0.069 & 0.130 & 0.012 \\
\hline 2012 & 0.090 & & 0.078 & -0.233 & 0.058 & & 0.332 & -0.551 & -0.351 & -0.508 & 0.189 & -0.104 & 0.204 & 0.060 \\
\hline 2013 & 0.122 & & 0.097 & -0.480 & 0.099 & & 0.596 & -0.092 & -0.579 & -0.577 & 0.258 & -0.027 & 0.273 & 0.099 \\
\hline
\end{tabular}

Source: Authors' calculation based on COMTRADE (2015) database 
Figure 13: Maturity level of brassieres and parts thereof (621210) at national and global level, 1996-2013

\begin{tabular}{|c|c|c|c|c|c|c|c|c|c|c|c|c|c|}
\hline Year & World & China & Egypt & Germany & Iran & Jordan & Lebanon & Morocco & S. Arabia & S. Korea & Tunisia & Turkey & USA \\
\hline 1996 & 0.069 & -0.131 & & 0.004 & & & & 0.759 & -1.083 & 0.032 & -0.057 & 0.191 & 0.073 \\
\hline 1997 & 0.066 & -0.208 & & 0.022 & 0.872 & -0.143 & 0.391 & 0.692 & & -0.032 & -0.028 & 0.212 & -0.004 \\
\hline 1998 & 0.054 & -0.285 & & 0.010 & 0.355 & 0.258 & 0.035 & 0.344 & -7.269 & -0.143 & -0.003 & 0.186 & -0.032 \\
\hline 1999 & 0.046 & -0.340 & & 0.003 & 0.028 & 0.417 & -0.195 & 0.134 & & -0.217 & 0.037 & 0.169 & -0.052 \\
\hline 2000 & 0.041 & -0.337 & & 0.002 & -0.344 & 0.329 & -0.323 & 0.003 & & -0.258 & 0.085 & 0.116 & -0.079 \\
\hline 2001 & 0.040 & -0.322 & 1.357 & -0.005 & -0.649 & -0.076 & -0.382 & -0.091 & -2.295 & -0.238 & 0.106 & 0.102 & -0.098 \\
\hline 2002 & 0.031 & -0.305 & & -0.007 & -0.594 & -0.347 & -0.354 & -0.169 & & -0.201 & 0.103 & 0.054 & -0.160 \\
\hline 2003 & 0.023 & -0.270 & 1.751 & 0.000 & -0.361 & & -0.307 & -0.237 & 4.963 & -0.220 & 0.085 & -0.014 & -0.242 \\
\hline 2004 & 0.016 & -0.248 & & 0.013 & -0.201 & -0.932 & -0.262 & -0.299 & & -0.244 & 0.059 & -0.075 & -0.301 \\
\hline 2005 & 0.010 & -0.180 & 2.898 & 0.026 & 0.404 & -1.297 & 0.026 & -0.262 & 7.525 & -0.236 & -0.003 & -0.153 & -0.321 \\
\hline 2006 & 0.008 & -0.138 & 3.683 & 0.041 & 0.574 & -1.537 & 0.156 & -0.133 & 4.388 & -0.207 & -0.038 & -0.198 & -0.302 \\
\hline 2007 & 0.004 & -0.165 & 3.001 & 0.056 & & -1.685 & 0.199 & -0.138 & 2.310 & -0.209 & -0.072 & -0.210 & -0.263 \\
\hline 2008 & 0.001 & -0.113 & 1.691 & 0.063 & & -1.829 & 0.229 & -0.183 & & -0.201 & -0.122 & -0.210 & -0.184 \\
\hline 2009 & 0.000 & -0.127 & 1.445 & 0.062 & & & 0.239 & -0.233 & & -0.172 & -0.202 & -0.189 & -0.051 \\
\hline 2010 & 0.001 & -0.141 & 0.992 & 0.049 & -0.123 & & 0.171 & -0.242 & & -0.170 & -0.210 & -0.170 & 0.120 \\
\hline 2011 & 0.003 & -0.165 & 0.581 & 0.025 & -0.898 & 0.176 & 0.085 & -0.238 & & -0.119 & -0.205 & -0.136 & 0.306 \\
\hline 2012 & 0.003 & -0.176 & 0.076 & -0.011 & & 0.824 & -0.018 & -0.216 & & -0.034 & -0.177 & -0.106 & 0.486 \\
\hline 2013 & 0.007 & -0.162 & -0.287 & -0.036 & & 1.027 & -0.290 & -0.208 & 5.468 & 0.023 & -0.092 & -0.081 & 0.608 \\
\hline
\end{tabular}

Source: Authors' calculation based on COMTRADE (2015) database 
Figure 14: Maturity level of articles of bedding nes (940490) at national and global level, 1996-2013

\begin{tabular}{|c|c|c|c|c|c|c|c|c|c|c|c|c|c|c|c|}
\hline Year & World & Algeria & Bahrain & China & Egypt & Germany & Jordan & Lebanon & Morocco & Oman & S. Arabia & S. Korea & Tunisia & Turkey & USA \\
\hline 1996 & 0.041 & -2.278 & & 0.025 & 0.031 & -0.050 & & & 0.603 & -0.547 & -0.340 & 0.431 & 0.187 & -0.107 & 0.056 \\
\hline 1997 & 0.073 & -0.994 & & 0.055 & -0.263 & -0.045 & 1.062 & 0.026 & 0.442 & & & 0.412 & 0.618 & -0.050 & 0.031 \\
\hline 1998 & 0.093 & -0.486 & & 0.060 & 0.343 & -0.042 & & 0.215 & 0.461 & 0.294 & -0.311 & 0.321 & 0.527 & 0.016 & 0.014 \\
\hline 1999 & 0.100 & -0.087 & & 0.042 & 0.580 & -0.030 & & 0.299 & 0.331 & 0.016 & -0.183 & 0.220 & 0.156 & 0.095 & 0.010 \\
\hline 2000 & 0.101 & 0.170 & -0.399 & 0.013 & 0.471 & -0.007 & 1.696 & 0.309 & 0.201 & -0.238 & -0.036 & 0.117 & -0.117 & 0.212 & 0.009 \\
\hline 2001 & 0.104 & 0.490 & -0.044 & 0.027 & 0.182 & 0.024 & 1.379 & 0.265 & 0.036 & -0.087 & 0.124 & 0.162 & -0.289 & 0.246 & -0.006 \\
\hline 2002 & 0.099 & 0.485 & 0.251 & 0.028 & 0.002 & 0.051 & 1.050 & 0.269 & -0.140 & 0.110 & 0.330 & 0.293 & -0.354 & 0.196 & -0.007 \\
\hline 2003 & 0.085 & 0.179 & 0.499 & 0.015 & -0.248 & 0.053 & 0.553 & 0.249 & -0.308 & 0.437 & 0.442 & 0.305 & -0.390 & 0.175 & -0.001 \\
\hline 2004 & 0.070 & 0.125 & 0.628 & 0.003 & -0.563 & 0.055 & 0.232 & 0.253 & -0.421 & 0.635 & 0.527 & 0.306 & -0.374 & 0.156 & -0.010 \\
\hline 2005 & 0.043 & 0.004 & 0.725 & -0.030 & -1.072 & 0.059 & -0.062 & 0.241 & -0.504 & 0.356 & 0.631 & 0.364 & -0.342 & 0.115 & -0.018 \\
\hline 2006 & 0.023 & 0.020 & 0.640 & -0.017 & -1.452 & 0.057 & -0.236 & 0.313 & -0.621 & 0.524 & 0.670 & 0.398 & -0.142 & 0.086 & -0.027 \\
\hline 2007 & 0.008 & 0.009 & 0.434 & -0.013 & -1.614 & 0.045 & -0.292 & 0.294 & -0.540 & 0.612 & 0.667 & 0.450 & -0.035 & 0.091 & -0.040 \\
\hline 2008 & -0.003 & 0.086 & 0.202 & -0.010 & -1.583 & 0.037 & -0.305 & 0.281 & -0.321 & 0.577 & & 0.509 & 0.120 & 0.065 & -0.048 \\
\hline 2009 & -0.001 & 0.210 & -0.236 & 0.011 & -1.130 & 0.033 & -0.280 & 0.285 & 0.038 & 0.143 & & 0.540 & 0.421 & -0.005 & -0.041 \\
\hline 2010 & -0.004 & 0.731 & -0.511 & 0.010 & -0.594 & 0.019 & -0.117 & 0.243 & 0.145 & -0.133 & 0.530 & 0.453 & 0.482 & -0.016 & -0.058 \\
\hline 2011 & -0.001 & 1.376 & -0.764 & 0.013 & -0.216 & 0.025 & 0.210 & 0.172 & 0.242 & -0.312 & 0.433 & 0.437 & 0.470 & -0.056 & -0.067 \\
\hline 2012 & 0.006 & 1.284 & -0.898 & 0.017 & 0.043 & 0.011 & 0.257 & 0.032 & 0.267 & -0.404 & 0.305 & 0.373 & 0.324 & -0.085 & -0.048 \\
\hline 2013 & 0.014 & 1.024 & -1.074 & 0.012 & 0.946 & 0.007 & 0.201 & 0.189 & 0.270 & -0.031 & -0.001 & 0.228 & -0.035 & -0.082 & -0.023 \\
\hline
\end{tabular}

Source: Authors' calculation based on COMTRADE (2015) database 
Figure 15: Maturity level of propene (propylene) (290122) at national and global level, 1996-2013

\begin{tabular}{|c|c|c|c|c|c|c|c|}
\hline Year & World & China & Germany & S. Arabia & S. Korea & Turkey & USA \\
\hline 1996 & -0.058 & 0.193 & -0.073 & 0.533 & 0.000 & 0.314 & -0.389 \\
\hline 1997 & -0.088 & -1.396 & -0.122 & & 0.018 & 1.822 & -0.328 \\
\hline 1998 & -0.067 & & -0.090 & 0.550 & 0.006 & 1.453 & -0.180 \\
\hline 1999 & -0.061 & -2.043 & -0.055 & 0.387 & -0.031 & 1.255 & -0.076 \\
\hline 2000 & -0.073 & -2.668 & -0.053 & 0.308 & -0.064 & 1.136 & 0.064 \\
\hline 2001 & -0.054 & -2.511 & -0.049 & 0.170 & -0.058 & 1.010 & 0.177 \\
\hline 2002 & -0.052 & -1.529 & 0.010 & 0.143 & -0.008 & 0.863 & 0.167 \\
\hline 2003 & -0.062 & -1.472 & 0.076 & -0.071 & 0.018 & & 0.182 \\
\hline 2004 & -0.068 & -1.383 & 0.122 & -0.195 & 0.033 & 0.650 & 0.172 \\
\hline 2005 & -0.066 & -0.450 & 0.147 & -0.273 & 0.059 & -0.048 & 0.114 \\
\hline 2006 & -0.078 & -0.614 & 0.140 & -0.314 & 0.101 & 0.085 & 0.041 \\
\hline 2007 & -0.070 & -0.843 & 0.099 & -0.335 & 0.143 & -0.085 & -0.049 \\
\hline 2008 & -0.045 & -0.622 & 0.026 & -0.249 & 0.136 & -0.042 & -0.189 \\
\hline 2009 & -0.005 & -0.286 & -0.009 & -0.035 & 0.112 & -0.082 & -0.210 \\
\hline 2010 & 0.011 & -0.605 & -0.090 & 0.056 & 0.090 & 0.056 & -0.233 \\
\hline 2011 & 0.027 & -0.445 & -0.180 & 0.184 & 0.083 & 0.184 & -0.283 \\
\hline 2012 & 0.036 & -0.470 & -0.231 & 0.222 & 0.101 & 0.366 & -0.279 \\
\hline 2013 & 0.050 & 0.155 & -0.193 & 0.002 & 0.115 & 0.664 & -0.217 \\
\hline
\end{tabular}

Source: Authors' calculation based on COMTRADE (2015) database 
Figure 16: Maturity level of polypropylene in primary forms (390210) at national and global level, 1996-2013

\begin{tabular}{|c|c|c|c|c|c|c|c|c|c|c|c|c|c|c|c|}
\hline Year & World & Bahrain & China & Egypt & Germany & Iran & Jordan & Lebanon & Morocco & Oman & S. Arabia & S. Korea & Tunisia & Turkey & USA \\
\hline 1996 & -0.086 & & -0.018 & -0.974 & 0.026 & & & & 4.125 & & -0.025 & -0.144 & 0.836 & -0.430 & -0.026 \\
\hline 1997 & -0.060 & & -0.305 & -0.866 & -0.066 & -0.235 & 0.891 & -1.205 & 1.175 & 2.746 & & -0.142 & 0.892 & -0.185 & 0.019 \\
\hline 1998 & -0.013 & & -0.419 & -0.308 & -0.090 & & & -1.174 & & 1.700 & 0.345 & -0.106 & 0.304 & -0.115 & 0.068 \\
\hline 1999 & 0.025 & & -0.382 & 0.055 & -0.124 & & 0.667 & -1.105 & -1.377 & 1.053 & 0.317 & -0.054 & 0.069 & -0.050 & 0.090 \\
\hline 2000 & 0.044 & -1.814 & -0.381 & 0.313 & -0.162 & -1.194 & 0.060 & -0.740 & -0.578 & & 0.268 & -0.027 & -0.088 & 0.021 & 0.115 \\
\hline 2001 & 0.060 & & -0.346 & 0.411 & -0.116 & -1.181 & -0.289 & -0.443 & 0.265 & & 0.173 & -0.021 & -0.240 & -0.096 & 0.119 \\
\hline 2002 & 0.065 & & -0.328 & 0.493 & -0.065 & -0.678 & -0.499 & -0.187 & 1.230 & 0.795 & 0.089 & -0.005 & -0.302 & 0.012 & 0.120 \\
\hline 2003 & 0.060 & & -0.313 & 0.546 & -0.050 & -0.083 & -0.666 & -0.094 & 2.217 & & 0.197 & -0.029 & -0.382 & 0.148 & 0.107 \\
\hline 2004 & 0.056 & -0.335 & -0.268 & 0.577 & -0.042 & 0.369 & -0.776 & -0.081 & & 1.234 & 0.318 & -0.054 & -0.427 & 0.243 & 0.091 \\
\hline 2005 & 0.037 & 0.099 & -0.018 & 0.448 & -0.003 & 0.658 & -0.781 & -0.167 & 3.945 & 1.615 & 0.429 & -0.095 & -0.442 & 0.236 & 0.050 \\
\hline 2006 & 0.014 & 0.482 & -0.010 & 0.249 & -0.010 & 1.190 & -0.774 & -0.231 & 5.028 & 1.804 & 0.527 & -0.128 & -0.143 & 0.260 & 0.011 \\
\hline 2007 & -0.004 & 1.268 & 0.048 & 0.215 & -0.031 & & -0.663 & -0.335 & 4.067 & 1.917 & 0.681 & -0.164 & -0.122 & 0.269 & -0.030 \\
\hline 2008 & -0.010 & 1.184 & 0.112 & 0.204 & -0.023 & & -0.458 & -0.397 & 2.713 & 1.959 & 0.825 & -0.165 & -0.106 & 0.238 & -0.084 \\
\hline 2009 & -0.003 & 0.582 & 0.170 & 0.179 & 0.012 & & -0.164 & -0.482 & 0.621 & -0.370 & 0.821 & -0.163 & -0.133 & 0.184 & -0.111 \\
\hline 2010 & 0.003 & 0.079 & 0.219 & 0.145 & 0.008 & 2.634 & 0.102 & -0.633 & -0.002 & -0.450 & 0.822 & -0.151 & 0.233 & 0.073 & -0.147 \\
\hline 2011 & 0.012 & -0.300 & 0.255 & 0.277 & 0.024 & 4.309 & 0.377 & -0.640 & -0.632 & -0.459 & 0.736 & -0.093 & 0.725 & -0.044 & -0.172 \\
\hline 2012 & 0.017 & -0.549 & 0.249 & 0.316 & 0.029 & & 0.646 & -0.517 & -0.874 & -0.507 & 0.584 & -0.006 & 1.217 & -0.121 & -0.195 \\
\hline 2013 & 0.027 & -1.354 & 0.218 & 0.384 & 0.062 & & 0.810 & -0.157 & -1.324 & -0.925 & 0.326 & 0.091 & 1.778 & -0.201 & -0.187 \\
\hline
\end{tabular}

Source: Authors' calculation based on COMTRADE (2015) database 
Figure 17: Maturity level of transistors, except photosensitive, $>1$ watt (854129) at national and global level, 1996-2013

\begin{tabular}{|c|c|c|c|c|c|c|c|c|c|c|c|}
\hline Year & World & China & Germany & Jordan & Lebanon & Morocco & S. Arabia & S. Korea & Tunisia & Turkey & USA \\
\hline 1996 & 0.061 & 0.422 & 0.058 & & & 1.622 & -0.098 & -0.254 & -0.168 & -0.156 & 0.028 \\
\hline 1997 & 0.063 & 0.250 & 0.093 & & & 1.439 & & -0.147 & -0.007 & 0.316 & 0.041 \\
\hline 1998 & 0.048 & 0.110 & 0.095 & & -0.509 & 0.832 & 0.250 & 0.001 & 0.578 & 0.388 & 0.030 \\
\hline 1999 & 0.026 & 0.070 & 0.087 & 0.165 & 0.034 & 0.477 & 0.450 & 0.098 & 0.154 & 0.499 & -0.009 \\
\hline 2000 & 0.001 & 0.080 & 0.040 & -0.787 & 0.718 & 0.225 & 0.671 & 0.166 & -0.752 & 0.507 & -0.032 \\
\hline 2001 & -0.010 & 0.065 & -0.010 & -1.188 & 0.959 & 0.058 & 0.705 & 0.316 & & 0.439 & -0.035 \\
\hline 2002 & -0.034 & 0.056 & -0.060 & -1.475 & 1.098 & -0.089 & 1.340 & 0.177 & & 0.321 & -0.028 \\
\hline 2003 & -0.053 & 0.074 & -0.097 & -1.145 & & -0.219 & 1.883 & 0.051 & -3.514 & 0.272 & 0.016 \\
\hline 2004 & -0.066 & 0.095 & -0.110 & & 1.085 & -0.323 & 2.170 & -0.057 & -4.028 & 0.257 & 0.062 \\
\hline 2005 & -0.075 & 0.206 & -0.140 & -1.442 & 0.910 & -0.255 & 2.308 & -0.226 & & 0.048 & 0.092 \\
\hline 2006 & -0.069 & 0.168 & -0.129 & & 0.872 & -0.047 & 2.546 & -0.339 & & 0.079 & 0.096 \\
\hline 2007 & -0.057 & 0.185 & -0.107 & & 0.581 & -0.056 & 2.719 & -0.427 & & 0.088 & 0.104 \\
\hline 2008 & -0.041 & 0.179 & -0.104 & -0.074 & 0.257 & -0.078 & & -0.476 & & -0.013 & 0.084 \\
\hline 2009 & -0.010 & 0.174 & -0.062 & 0.869 & -0.233 & -0.096 & & -0.489 & & -0.074 & 0.026 \\
\hline 2010 & 0.020 & 0.190 & -0.001 & & -0.492 & -0.079 & & -0.406 & 1.100 & -0.052 & -0.055 \\
\hline 2011 & 0.042 & 0.196 & 0.043 & & -0.532 & -0.063 & & -0.317 & 0.641 & -0.095 & -0.186 \\
\hline 2012 & 0.062 & 0.208 & 0.066 & & -0.785 & -0.059 & & -0.138 & -0.240 & -0.149 & -0.328 \\
\hline 2013 & 0.090 & 0.242 & 0.118 & & -1.327 & -0.048 & & 0.123 & -0.637 & -0.089 & -0.396 \\
\hline
\end{tabular}

Source: Authors' calculation based on COMTRADE (2015) database 
Figure 18: Maturity level of pneumatic tyres new of rubber for buses or lorries (401120) at national and global level, 1996-2013

\begin{tabular}{|c|c|c|c|c|c|c|c|c|c|c|c|c|c|c|c|c|}
\hline Year & World & Algeria & Bahrain & China & Egypt & Germany & Iran & Jordan & Lebanon & Morocco & Oman & S. Arabia & S. Korea & Tunisia & Turkey & USA \\
\hline 1996 & -0.013 & -2.666 & & 0.136 & 0.380 & -0.024 & & & & -0.290 & 0.186 & -1.168 & -0.002 & -0.466 & 0.128 & 0.050 \\
\hline 1997 & -0.029 & -2.271 & & 0.157 & 0.393 & -0.026 & 0.821 & -0.359 & 0.039 & -0.076 & 0.013 & & -0.044 & -0.134 & 0.142 & -0.022 \\
\hline 1998 & -0.040 & -0.926 & & 0.136 & 0.488 & -0.033 & 0.673 & & 0.303 & 0.147 & -0.182 & 0.078 & -0.053 & 0.115 & 0.157 & -0.067 \\
\hline 1999 & -0.032 & 0.560 & & 0.088 & 0.256 & -0.038 & 0.450 & -0.275 & 0.146 & 0.293 & -0.420 & 0.232 & -0.039 & 0.299 & 0.164 & -0.074 \\
\hline 2000 & -0.014 & 1.342 & 0.116 & 0.045 & 0.113 & -0.022 & 0.234 & -0.323 & 0.029 & 0.200 & -0.521 & 0.421 & -0.015 & 0.444 & 0.167 & -0.053 \\
\hline 2001 & 0.003 & & 0.324 & 0.048 & -0.008 & -0.003 & 0.130 & -0.305 & -0.041 & 0.082 & -0.476 & 0.437 & -0.015 & 0.508 & 0.142 & -0.037 \\
\hline 2002 & 0.008 & 1.939 & 0.505 & 0.048 & -0.075 & -0.002 & -0.015 & -0.296 & -0.012 & -0.081 & -0.372 & 0.491 & -0.007 & 0.554 & 0.113 & -0.017 \\
\hline 2003 & 0.003 & 1.840 & 0.761 & 0.034 & -0.086 & -0.011 & -0.122 & -0.320 & 0.035 & -0.237 & -0.288 & 0.536 & -0.009 & 0.654 & 0.021 & 0.002 \\
\hline 2004 & 0.001 & 1.712 & 0.883 & 0.027 & -0.141 & -0.014 & -0.212 & -0.349 & 0.067 & -0.363 & -0.219 & 0.526 & -0.008 & 0.773 & -0.025 & 0.015 \\
\hline 2005 & 0.003 & 1.261 & 0.955 & -0.004 & -0.146 & -0.013 & -0.333 & -0.332 & -0.057 & -0.519 & -0.144 & 0.391 & -0.001 & 0.697 & -0.064 & 0.045 \\
\hline 2006 & 0.003 & 0.725 & 0.964 & -0.003 & -0.114 & -0.011 & -0.155 & -0.369 & -0.151 & -0.730 & -0.143 & 0.426 & 0.001 & 0.662 & -0.078 & 0.061 \\
\hline 2007 & -0.007 & 0.195 & 0.900 & -0.008 & 0.143 & -0.018 & & -0.339 & -0.164 & -0.779 & -0.165 & 0.473 & -0.006 & 0.616 & -0.074 & 0.062 \\
\hline 2008 & -0.018 & -0.044 & 0.765 & 0.003 & 0.280 & -0.018 & & -0.224 & -0.170 & -0.395 & -0.244 & & -0.010 & 0.461 & -0.056 & 0.051 \\
\hline 2009 & -0.007 & -0.325 & 0.491 & 0.036 & 0.424 & -0.015 & & -0.020 & -0.202 & 0.241 & -0.542 & & -0.008 & 0.189 & -0.023 & 0.056 \\
\hline 2010 & 0.009 & -0.398 & 0.237 & 0.051 & 0.570 & -0.003 & 2.008 & 0.004 & -0.172 & 0.369 & -0.787 & 0.193 & 0.004 & 0.100 & 0.055 & 0.053 \\
\hline 2011 & 0.029 & -0.333 & -0.071 & 0.072 & 0.658 & 0.009 & 3.432 & 0.033 & -0.084 & 0.493 & -0.639 & 0.145 & 0.025 & -0.043 & 0.185 & 0.055 \\
\hline 2012 & 0.043 & -0.390 & -0.174 & 0.088 & 0.689 & 0.007 & & 0.079 & -0.005 & 0.587 & -0.470 & 0.105 & 0.046 & -0.181 & 0.225 & 0.059 \\
\hline 2013 & 0.053 & -0.491 & -0.478 & 0.086 & 0.683 & 0.001 & & 0.097 & -0.058 & 0.561 & -0.215 & 0.246 & 0.040 & -0.106 & 0.228 & 0.040 \\
\hline
\end{tabular}

Source: Authors' calculation based on COMTRADE (2015) database 
Figure 19: Maturity level of polyethylene - specific gravity $>0.94$ in primary form (390120) at national and global level, 1996-2013

\begin{tabular}{|c|c|c|c|c|c|c|c|c|c|c|c|c|c|c|c|}
\hline Year & World & Bahrain & China & Egypt & Germany & Iran & Jordan & Kuwait & Lebanon & Morocco & S. Arabia & S. Korea & Tunisia & Turkey & USA \\
\hline 1996 & -0.011 & & -0.480 & & 0.098 & & & & & & -0.006 & 0.002 & 0.765 & 0.042 & -0.119 \\
\hline 1997 & -0.021 & & -1.009 & -3.418 & 0.005 & -1.185 & & & -0.944 & & & -0.065 & 0.118 & 0.301 & -0.061 \\
\hline 1998 & -0.012 & & -1.179 & -0.867 & -0.023 & & & & & & 0.293 & -0.070 & -0.195 & 0.421 & -0.011 \\
\hline 1999 & -0.001 & & -1.127 & & -0.041 & & 0.717 & & 0.417 & -1.492 & 0.204 & -0.055 & -0.208 & 0.445 & 0.020 \\
\hline 2000 & 0.004 & 5.959 & -1.085 & 1.409 & -0.052 & 2.665 & 0.814 & -0.077 & 0.273 & & 0.131 & -0.043 & 0.311 & 0.371 & 0.032 \\
\hline 2001 & 0.010 & & -0.967 & 1.778 & -0.064 & 1.498 & 0.834 & 0.176 & 0.212 & 1.290 & 0.015 & -0.055 & 0.841 & 0.166 & 0.049 \\
\hline 2002 & 0.027 & 2.342 & -0.818 & 2.101 & -0.021 & 0.948 & 0.758 & 0.454 & 0.286 & 2.064 & -0.046 & 0.000 & 0.993 & 0.151 & 0.079 \\
\hline 2003 & 0.032 & 1.378 & -0.780 & 2.257 & 0.014 & 0.649 & 0.557 & 0.653 & 0.247 & 2.806 & -0.090 & 0.028 & 1.060 & 0.206 & 0.110 \\
\hline 2004 & 0.028 & 0.671 & -0.730 & 2.447 & 0.027 & 0.444 & 0.447 & 0.796 & 0.112 & 3.296 & -0.122 & 0.035 & 1.041 & 0.280 & 0.130 \\
\hline 2005 & 0.020 & & -0.296 & 1.787 & 0.064 & 0.239 & 0.239 & & 0.139 & 3.049 & -0.194 & 0.043 & 1.184 & 0.273 & 0.136 \\
\hline 2006 & 0.011 & 0.323 & -0.385 & 1.223 & 0.063 & -0.203 & 0.164 & 0.967 & 0.081 & 2.728 & -0.217 & 0.034 & 1.148 & 0.371 & 0.127 \\
\hline 2007 & -0.002 & 0.773 & -0.280 & 1.019 & 0.032 & & 0.141 & 1.055 & -0.113 & 2.392 & -0.237 & 0.017 & 1.006 & 0.396 & 0.122 \\
\hline 2008 & -0.010 & 0.772 & -0.177 & 0.524 & -0.002 & & 0.204 & 1.155 & -0.319 & 2.157 & -0.178 & 0.004 & 0.748 & 0.327 & 0.115 \\
\hline 2009 & -0.007 & 0.407 & -0.070 & 0.276 & -0.025 & & 0.281 & 1.316 & -0.442 & 0.992 & -0.094 & -0.019 & 0.032 & 0.245 & 0.077 \\
\hline 2010 & -0.015 & -0.244 & -0.032 & 0.005 & -0.060 & -0.539 & 0.483 & & -0.448 & 0.756 & -0.050 & -0.054 & 0.142 & 0.188 & 0.026 \\
\hline 2011 & -0.008 & -0.983 & 0.053 & -0.083 & -0.071 & -1.321 & 0.721 & & -0.245 & 0.342 & -0.014 & -0.057 & 0.265 & 0.131 & -0.023 \\
\hline 2012 & 0.015 & -1.877 & 0.101 & -0.203 & -0.047 & & 0.801 & & 0.095 & 0.081 & 0.046 & -0.019 & 0.468 & 0.020 & -0.066 \\
\hline 2013 & 0.050 & -2.857 & 0.104 & -0.401 & -0.015 & & 0.692 & 0.069 & 0.505 & -0.310 & 0.083 & 0.041 & 0.578 & -0.178 & -0.106 \\
\hline
\end{tabular}

Source: Authors' calculation based on COMTRADE (2015) database 
Figure 20: Maturity level of sacks and bags (including cones) of polymers of ethylen (392321) at national and global level, $1996-2013$

\begin{tabular}{|c|c|c|c|c|c|c|c|c|c|c|c|c|c|c|c|c|}
\hline Year & World & Algeria & Bahrain & China & Egypt & Germany & Iran & Jordan & Lebanon & Morocco & Oman & S. Arabia & S. Korea & Tunisia & Turkey & USA \\
\hline 1996 & 0.021 & & & 0.088 & -0.205 & -0.044 & & & & -0.494 & 0.605 & 0.161 & 0.122 & 0.963 & -0.172 & 0.097 \\
\hline 1997 & 0.027 & & & 0.080 & -0.517 & -0.021 & 0.798 & -0.072 & 0.220 & -0.452 & 0.472 & & 0.032 & 1.286 & -0.220 & 0.087 \\
\hline 1998 & 0.039 & & & 0.081 & -0.135 & 0.017 & 0.549 & & 0.142 & -0.190 & 0.159 & 0.249 & 0.002 & 1.523 & -0.131 & 0.067 \\
\hline 1999 & 0.053 & & & 0.095 & 0.116 & 0.059 & 0.444 & 0.299 & 0.179 & -0.183 & -0.068 & 0.353 & -0.015 & 1.540 & -0.029 & 0.063 \\
\hline 2000 & 0.062 & 0.281 & 0.250 & 0.088 & 0.101 & 0.100 & 0.287 & 0.865 & 0.152 & -0.188 & -0.158 & 0.415 & -0.028 & 1.184 & 0.026 & 0.078 \\
\hline 2001 & 0.062 & & 0.193 & 0.065 & -0.007 & 0.110 & 0.087 & & 0.109 & -0.098 & -0.099 & 0.475 & -0.002 & 0.914 & 0.052 & 0.081 \\
\hline 2002 & 0.067 & -1.362 & 0.103 & 0.064 & -0.099 & 0.125 & 0.227 & 1.278 & 0.111 & -0.027 & 0.078 & 0.437 & 0.001 & 0.757 & 0.085 & 0.079 \\
\hline 2003 & 0.066 & -1.063 & -0.027 & 0.057 & -0.152 & 0.134 & 0.450 & 1.271 & 0.113 & 0.143 & 0.344 & 0.751 & -0.007 & 0.685 & 0.106 & 0.074 \\
\hline 2004 & 0.062 & & -0.123 & 0.042 & -0.210 & 0.137 & 0.717 & 1.145 & 0.135 & 0.291 & 0.366 & 0.659 & -0.013 & 0.598 & 0.116 & 0.066 \\
\hline 2005 & 0.048 & & -0.195 & 0.023 & -0.316 & 0.119 & 0.793 & 0.841 & 0.191 & 0.407 & 0.538 & 0.595 & -0.008 & 0.414 & 0.127 & 0.053 \\
\hline 2006 & 0.035 & -0.216 & -0.145 & 0.001 & -0.521 & 0.096 & 0.756 & 0.630 & 0.271 & 0.380 & 0.591 & 0.591 & -0.008 & 0.322 & 0.077 & 0.045 \\
\hline 2007 & 0.020 & 0.434 & -0.126 & -0.024 & -0.571 & 0.076 & & 0.326 & 0.272 & 0.530 & 0.564 & 0.605 & -0.005 & 0.298 & 0.057 & 0.026 \\
\hline 2008 & 0.008 & & -0.043 & -0.048 & -0.542 & 0.050 & & -0.054 & 0.226 & 0.556 & 0.447 & & 0.008 & 0.220 & 0.029 & -0.003 \\
\hline 2009 & -0.003 & 1.816 & 0.118 & -0.064 & -0.269 & 0.017 & & -0.649 & 0.149 & 0.540 & 0.055 & & 0.034 & 0.329 & 0.009 & -0.004 \\
\hline 2010 & -0.011 & 1.094 & 0.318 & -0.079 & 0.072 & -0.002 & -0.926 & -0.746 & 0.090 & 0.384 & -0.524 & 0.068 & 0.052 & 0.313 & 0.006 & -0.009 \\
\hline 2011 & -0.010 & -0.080 & 0.544 & -0.085 & 0.301 & -0.010 & -2.114 & -0.673 & 0.033 & 0.066 & -0.682 & 0.036 & 0.078 & 0.356 & 0.012 & -0.010 \\
\hline 2012 & -0.001 & -1.395 & 0.699 & -0.077 & 0.587 & -0.010 & & -0.392 & -0.031 & -0.309 & -0.979 & 0.385 & 0.118 & 0.427 & 0.034 & -0.001 \\
\hline 2013 & 0.021 & & 0.634 & -0.047 & 0.540 & 0.009 & & -0.075 & -0.065 & -0.594 & -1.147 & 1.084 & 0.182 & 0.070 & 0.075 & 0.026 \\
\hline
\end{tabular}

Source: Authors' calculation based on COMTRADE (2015) database 